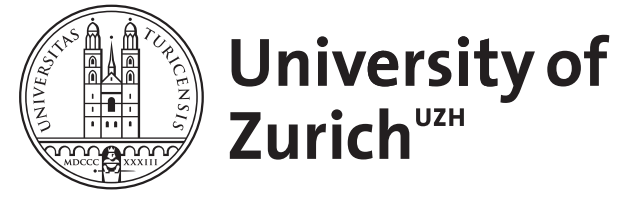

Zurich Open Repository and Archive

University of Zurich

University Library

Strickhofstrasse 39

CH-8057 Zurich

www.zora.uzh.ch

Year: 2008

\title{
A general technique to prove upper bounds for singular perturbation problems
}

Poliakovsky, A

DOI: https://doi.org/10.1007/s11854-008-0024-6

Posted at the Zurich Open Repository and Archive, University of Zurich ZORA URL: https://doi.org/10.5167/uzh-21455

Journal Article

Published Version

Originally published at:

Poliakovsky, A (2008). A general technique to prove upper bounds for singular perturbation problems. Journal d'Analyse Mathématique, 104:247-290.

DOI: https://doi.org/10.1007/s11854-008-0024-6 


\title{
A GENERAL TECHNIQUE TO PROVE UPPER BOUNDS FOR SINGULAR PERTURBATION PROBLEMS
}

\author{
By \\ ARKADY POLIAKOVSKY
}

\section{Introduction}

1.1 The motivation and the main results. Let $\mathcal{T}$ be a metric space. When $\varepsilon \rightarrow 0$, the asymptotic behavior of the family $\left\{I_{\varepsilon}\right\}_{\varepsilon>0}$ of the functionals $I_{\varepsilon}(\phi): \mathcal{T} \rightarrow \mathbb{R}^{+} \cup\{0\} \cup\{+\infty\}$ is partially described by De Giorgi's $\Gamma$-limit

$$
I_{0}(\phi):=\inf _{\{\varepsilon\},\left\{\phi_{\varepsilon}\right\}}\left\{\varliminf_{\varepsilon \rightarrow 0^{+}} I_{\varepsilon}\left(\phi_{\varepsilon}\right): \phi_{\varepsilon} \rightarrow \phi \text { in } \mathcal{T}\right\} .
$$

Usually, to find the $\Gamma$-limit of $I_{\varepsilon}(\phi)$, we need to find two bounds.

* First, we wish to find a lower bound, i.e., a functional $\underline{I}(\phi)$ such that for each family $\left\{\phi_{\varepsilon}\right\}_{\varepsilon>0}$, satisfying $\phi_{\varepsilon} \rightarrow \phi$ as $\varepsilon \rightarrow 0^{+}$, we have $\underline{\lim }_{\varepsilon \rightarrow 0^{+}} I_{\varepsilon}\left(\phi_{\varepsilon}\right) \geq \underline{I}(\phi)$.

** Second, we wish to find an upper bound, i.e., a functional $\bar{I}(\phi)$ such that there exists the family $\left\{\psi_{\varepsilon}\right\}_{\varepsilon>0}$, satisfying $\psi_{\varepsilon} \rightarrow \phi$ as $\varepsilon \rightarrow 0^{+}$, and we have $\varlimsup_{\varepsilon \rightarrow 0^{+}} I_{\varepsilon}\left(\psi_{\varepsilon}\right) \leq \bar{I}(\phi)$.

**** If we obtain $\underline{I}(\phi)=\bar{I}(\phi):=I(\phi)$, then $I(\phi)$ is the $\Gamma$-limit of $I_{\varepsilon}(\phi)$.

In various applications, we deal with the asymptotic behavior of a family of functionals of first and second order. In the case of first order problems, the functional $I_{\varepsilon}$, which acts on scalar or vector-valued functions $\phi$, has the form

$$
I_{\varepsilon}(\phi)=\int_{\Omega} \varepsilon|\nabla \phi(x)|^{2}+\frac{1}{\varepsilon} W(\phi(x), x) d x
$$

or, more generally,

$$
I_{\varepsilon}(\phi)=\int_{\Omega} \frac{1}{\varepsilon} L(\varepsilon \nabla \phi(x), \phi(x), x) d x .
$$

In the case of second order problems, the functional $I_{\varepsilon}$, which acts on scalar or vector-valued functions $v$, has the form

$$
I_{\varepsilon}(v)=\int_{\Omega} \varepsilon\left|\nabla^{2} v(x)\right|^{2}+\frac{1}{\varepsilon} W(\nabla v(x), v(x), x) d x
$$


or, more generally,

$$
I_{\varepsilon}(v)=\int_{\Omega} \frac{1}{\varepsilon} L\left(\varepsilon \nabla^{2} v(x), \nabla v(x), v(x), x\right) d x .
$$

Functionals of the form (1.1) arise in the theories of phase transitions and minimal surfaces. They were first studied by Modica and Mortola [20], Modica [18], Sternberg [24] and others. The $\Gamma$-limit of the functional in (1.1), where $W$ does not depend on $x$ explicitly, was obtained in the general vectorial case by Ambrosio in [1]. For an example of problem (1.1) with singular explicit dependence of $W$ on $x$, we can mention here the functional

$$
\int_{\Omega} \varepsilon|\nabla \phi(x)|^{2}+\frac{1}{\varepsilon}\left|e^{i \phi(x)}-u(x)\right|^{p} d x,
$$

where $u \in B V\left(\Omega, S^{1}\right)$ and $p>1$, related to the problem of the optimal $B V$-lifting. See [22], where we find its $\Gamma$ limit.

The functionals of second order of the form (1.3) arise, for example, in the gradient theory of solid-solid phase transitions, where one considers energies of the form

$$
I_{\varepsilon}(v)=\int_{\Omega} \varepsilon\left|\nabla^{2} v(x)\right|^{2}+\frac{1}{\varepsilon} W(\nabla v(x)) d x,
$$

where $v: \Omega \subset \mathbb{R}^{N} \rightarrow \mathbb{R}^{N}$ stands for the deformation, and the free energy density $W(F)$ is non-negative and satisfies

$$
W(F)=0 \quad \text { if and only if } \quad F \in K:=S O(N) A \cap S O(N) B .
$$

Here $A$ and $B$ are two fixed, invertible matrices such that $\operatorname{rank}(A-B)=1$, and $S O(N)$ is the set of rotations in $\mathbb{R}^{N}$. The simpler case in which $W(F)=0$ if and only if $F \in\{A, B\}$ was studied by Conti, Fonseca and Leoni in [8]. The case of problem (1.5), where $N=2$ and $W(Q F)=W(F)$ for all $Q \in S O(2)$, was investigated by Conti and Schweizer in [7] (see also [6] for a related problem). Another important example of the second order energy is the so called Aviles-Giga functional, defined on scalar valued functions $v$ by

$$
\int_{\Omega} \varepsilon\left|\nabla^{2} v\right|^{2}+\frac{1}{\varepsilon}\left(1-|\nabla v|^{2}\right)^{2} \quad \text { (see [2]). }
$$

The main contribution of this work is to give a new method for finding upper bounds in the sense of (**) for families of functionals of the first and second order. When the functionals are of the form (1.1) or (1.2), the convergence is in the $L^{p}$-sense; and when they are of the form (1.3) or (1.4), it is in the $W^{1, p}$-sense. 
What can we expect as reasonable upper bounds? It is clear that if the nonnegative function $W$ is continuous in the argument $\phi$, then every upper bound $\bar{I}(\phi)$ of (1.1) will be finite only if $W(\phi, x)=0$ a.e. More generally, if the non-negative function $L$ is continuous in the variable $\phi$ and satisfies $L(0, \phi, x) \leq L(\nabla \phi, \phi, x)$, then every upper bound $\bar{I}(\phi)$ of (1.2) will be finite only if $L(0, \phi, x)=0$ a.e. The same holds for the second order problem: if the non-negative function $L$ is continuous in the variables $\nabla v$ and $v$ and satisfies $L(0, \nabla v, v, x) \leq L\left(\nabla^{2} v, \nabla v, v, x\right)$, then every upper bound $\bar{I}(v)$ of (1.4) will be finite only if $L(0, \nabla v, v, x)=0$ a.e. On the other hand, we prove the following theorem about first order problems.

Theorem 1.1. Let $\Omega \subset \mathbb{R}^{N}$ be a bounded Lipschitz domain and let $H(a, b, c)$ : $\mathbb{R}^{d \times N} \times \mathbb{R}^{d} \times \mathbb{R}^{q} \rightarrow \mathbb{R}$ be a $C^{1}$-function satisfying $H(a, b, c) \geq 0$ for every $a \in \mathbb{R}^{d \times N}$, $b \in \mathbb{R}^{d}$ and $c \in \mathbb{R}^{q}$. Consider $u \in B V\left(\Omega, \mathbb{R}^{q}\right) \cap L^{\infty}\left(\Omega, \mathbb{R}^{q}\right)$ and $\varphi \in B V\left(\Omega, \mathbb{R}^{d}\right) \cap$ $L^{\infty}\left(\Omega, \mathbb{R}^{d}\right)$ satisfying

$$
H(O, \varphi(x), u(x))=0 \quad \text { for a.e. } x \in \Omega .
$$

Then there exists a family of functions $\left\{\psi_{\varepsilon}\right\} \subset C^{2}\left(\mathbb{R}^{N}, \mathbb{R}^{d}\right), 0<\varepsilon<1$, such that $\int_{\Omega} \psi_{\varepsilon}(x) d x=\int_{\Omega} \varphi(x) d x$,

$$
\psi_{\varepsilon}(x) \rightarrow \varphi(x) \text { in } L^{p}\left(\Omega, \mathbb{R}^{d}\right) \quad \text { for all } p \in[1, \infty),
$$

and

$$
\begin{aligned}
\lim _{\varepsilon \rightarrow 0^{+}} & \int_{\Omega} \frac{1}{\varepsilon} H\left(\varepsilon \nabla \psi_{\varepsilon}, \psi_{\varepsilon}, u\right) d x=\mathcal{J}_{0}(\varphi) \\
:= & \int_{J_{\varphi}}\left(\operatorname { i n f } _ { r \in \mathcal { R } _ { \varphi ^ { + } ( x ) , \varphi ^ { - } ( x ) } ^ { ( 0 ) } } \left\{\int_{-\infty}^{0} H\left(-r^{\prime}(t) \otimes \boldsymbol{\nu}(x), r(t), u^{+}(x)\right) d t\right.\right. \\
& \left.\left.+\int_{0}^{\infty} H\left(-r^{\prime}(t) \otimes \boldsymbol{\nu}(x), r(t), u^{-}(x)\right) d t\right\}\right) d \mathcal{H}^{N-1}(x),
\end{aligned}
$$

where $\mathcal{R}_{a, b}^{(0)}$ is defined by

$$
\mathcal{R}_{\boldsymbol{a}, \boldsymbol{b}}^{(0)}:=\left\{r(t) \in C^{1}\left(\mathbb{R}, \mathbb{R}^{d}\right):\right.
$$

$$
\exists L>0 \text { s.t. } r(t)=\boldsymbol{a} \text { for } t \leq-L, r(t)=\boldsymbol{b} \text { for } t \geq L\},
$$

$J_{\varphi}$ is the jump set of $\varphi$ (see Definition 2.2) and we assume that the orientation of $J_{u}$ coincides with the orientation of $J_{\varphi} \mathcal{H}^{N-1}$ a.e. on $J_{u} \cap J_{\varphi}$.

We also generalize this result to a wider class of functionals; see Theorem 5.2. As for the second order problems, using a similar method, we prove the following theorem. 
Theorem 1.2. Let $\Omega$ be a bounded $C^{2}$-domain and let

$$
F(a, b, c, d): \mathbb{R}^{d \times N \times N} \times \mathbb{R}^{d \times N} \times \mathbb{R}^{d} \times \mathbb{R}^{q} \rightarrow \mathbb{R}
$$

be a $C^{1}$ function satisfying $F \geq 0$. Let $f \in B V\left(\Omega, \mathbb{R}^{q}\right) \cap L^{\infty}\left(\Omega, \mathbb{R}^{q}\right)$ and $v \in W^{1, \infty}\left(\Omega, \mathbb{R}^{d}\right)$ be such that $\nabla v \in B V\left(\Omega, \mathbb{R}^{d \times N}\right)$ and $F(0, \nabla v(x), v(x), f(x))=0$ a.e. in $\Omega$. Then there exists a family of functions $\left\{v_{\varepsilon}\right\} \subset C^{2}\left(\mathbb{R}^{N}, \mathbb{R}^{d}\right)$ satisfying

$$
v_{\varepsilon}(x) \rightarrow v(x) \quad \text { in } \quad W^{1, p}\left(\Omega, \mathbb{R}^{d}\right) \quad \text { for all } p \in[1, \infty),
$$

and

$$
\begin{aligned}
& \lim _{\varepsilon \rightarrow 0} \frac{1}{\varepsilon} \int_{\Omega} F\left(\varepsilon \nabla^{2} v_{\varepsilon}(x), \nabla v_{\varepsilon}(x), v_{\varepsilon}(x), f(x)\right) d x=\widetilde{\mathcal{J}}_{0}(v) \\
:= & \int_{J_{\nabla v}}\left(\operatorname { i n f } _ { r \in \mathcal { R } _ { \chi ( x ) , 0 } ^ { ( 0 ) } } \left\{\int_{-\infty}^{0} F\left(-r^{\prime}(t) \otimes \boldsymbol{\nu}(x) \otimes \boldsymbol{\nu}(x), r(t) \otimes \boldsymbol{\nu}(x)+\nabla v^{-}(x), v(x), f^{+}(x)\right) d t\right.\right. \\
+ & \left.\left.\int_{0}^{\infty} F\left(-r^{\prime}(t) \otimes \boldsymbol{\nu}(x) \otimes \boldsymbol{\nu}(x), r(t) \otimes \boldsymbol{\nu}(x)+\nabla v^{-}(x), v(x), f^{-}(x)\right) d t\right\}\right) d \mathcal{H}^{N-1}(x) .
\end{aligned}
$$

Here $\chi(x)$ is given by

$$
\chi(x) \otimes \boldsymbol{\nu}(x)=\nabla v^{+}(x)-\nabla v^{-}(x),
$$

$\mathcal{R}_{\boldsymbol{a}, \boldsymbol{b}}^{(0)}$ is defined by

$\mathcal{R}_{\boldsymbol{a}, \boldsymbol{b}}^{(0)}:=\left\{r(t) \in C^{1}\left(\mathbb{R}, \mathbb{R}^{d}\right): \exists L>0\right.$ s.t. $r(t)=\boldsymbol{a}$ for $t \leq-L, r(t)=\boldsymbol{b}$ for $\left.t \geq L\right\}$,

$J_{\nabla v}$ is the jump set of $\nabla v$ and we assume that the orientation of $J_{f}$ coincides with the orientation of $J_{\nabla v} \mathcal{H}^{N-1}$ a.e. on $J_{f} \cap J_{\nabla v}$.

In these theorems, we restrict ourselves to the case of $\varphi \in B V$ or $\nabla v \in B V$ (for first and second problems, respectively). But in general, for these problems, the limiting spaces, i.e., the spaces where the $\Gamma$-limits are finite, is wider then $B V$. Consider, for instance, the second order problem (1.6). Here constructing the upper bounds in full generality is a challenging unsolved question. A second question is whether the bounds of Theorem 1.1 and Theorem 1.2 are sharp. For first order problems, in all cases for which the $\Gamma$-limit is known, our method gives the sharp upper bound. As for second order problems, there exist examples, where our bound is far from being sharp. This happens in the cases in which the one-dimensional ansatz is no longer true. Since our method is based on convolutions with smoothing kernels, for functions which depend only on one variable, it gives an approximating sequence which asymptotically also depend 
on one variable. When the one-dimensional ansatz is valid, i.e., when for the function $\phi$ which depends only on one spatial variable we can consider an optimal asymptotic sequence that depends also on one variable, our method gives the sharp upper bound. For example, our upper bound is sharp for (1.6) in dimension $N=2$. In this case, the lower bound is known and coincides with our upper bound; see [23], [9] and [2]. For another example, we can take the functional in (1.5) where $W(F)=0$ if and only if $F \in\{A, B\}$ and $W$ satisfies the additional hypothesis, called $\left(H_{3}\right)$ in [8], which guarantees the validity of the one-dimensional ansatz in this case. However, at least in some cases, the one-dimensional ansatz (and, with it, the method presented in this paper) fails. For instance, for (1.6) in dimension $N>2$, the functional $\widetilde{\mathcal{J}}_{0}$ obtained in Theorem 1.2, is not lower-semi-continuous and therefore cannot be the $\Gamma$-limit; see [10]. The same holds for the problem

$$
\int_{\Omega} \varepsilon\left|\nabla^{2} v\right|^{2}+\frac{1}{\varepsilon}\left(1-|\nabla v|^{2}\right)^{p}
$$

where $N=2$ and $p>2$; see [2]. In [8] and [6], there are examples of vector-valued second order problems in which one-dimensional transition layers do not give the sharp bound but gives the same bound as our method. Constructing bounds which are sharper than ours is another open question. However, even in cases in which our method does not give the sharp upper bound, we get estimates of some interest. The important feature of the method is that it does not depend on subtle geometric ad hoc considerations depending on the particular variational problem. We also remark that most of the literature on singularly perturbed double-well potentials deal with asymptotic problems in which the one-dimensional hypothesis is valid. In these cases, our method succeeds in giving the sharp bound.

1.2 The strategy of the proof. Our main technique is a mollification of the vector-valued function with matrix-valued kernels. First of all, given a domain $\Omega \subset \mathbb{R}^{N}$, we define a special class of mollifying kernels $\mathcal{V}$ (the class of all smooth compactly supported $d \times d$-matrix valued functions $\eta$ in variables $(z, x) \in \mathbb{R}^{N} \times \mathbb{R}^{N}$, satisfying

$$
\int_{\mathbb{R}^{N}} \eta(z, x) d z=I \quad \text { for all } x \in \Omega
$$

where $I$ is the identity matrix; see Definition 4.1). Next, given $H, u$ and $\varphi$ as in Theorem 1.1 , for every $\eta \in \mathcal{V}$ we define

$$
\psi_{\varepsilon}(x):=\frac{1}{\varepsilon^{N}} \int_{\mathbb{R}^{N}} \eta\left(\frac{y-x}{\varepsilon}, x\right) \cdot \bar{\varphi}(y) d y,
$$

where $\bar{\varphi}$ is some $B V$-extension of $\varphi$ having no jump on $\partial \Omega$. Then we calculate

$$
Z(\eta):=\lim _{\varepsilon \rightarrow 0^{+}} \int_{\Omega} \frac{1}{\varepsilon} H\left(\varepsilon \nabla \psi_{\varepsilon}, \psi_{\varepsilon}, u\right) d x
$$


see Theorem 4.1. Next we optimize the choice of $\eta$, i.e., we calculate

$$
\mathcal{J}_{0}(\varphi):=\inf _{\eta \in \mathcal{V}} Z(\eta)
$$

see Theorem 5.1. Finally, we prove Theorem 1.1 by a classical diagonal argument.

We follow the same strategy in the case of the second order problem. Given $F$, $f$ and $v$ as in Theorem 1.2, we define

$$
v_{\varepsilon}(x):=\frac{1}{\varepsilon^{N}} \int_{\mathbb{R}^{N}} \eta\left(\frac{y-x}{\varepsilon}, x\right) \cdot \bar{v}(y) d y,
$$

where $\bar{v}$ is some extension of $v$ such that $\nabla \bar{v}$ has no jump on $\partial \Omega$. Then we calculate

$$
\tilde{Y}(\eta):=\lim _{\varepsilon \rightarrow 0} \frac{1}{\varepsilon} \int_{\Omega} F\left(\varepsilon \nabla^{2} v_{\varepsilon}(x), \nabla v_{\varepsilon}(x), v_{\varepsilon}(x), f(x)\right) d x ;
$$

see Theorem 6.1. In the proof, we easily reduce this problem to the previous first order problem. Next we calculate, as before,

$$
\widetilde{\mathcal{J}}_{0}(v):=\inf _{\eta \in \mathcal{V}} \tilde{Y}(\eta)
$$

Finally, we prove Theorem 1.2 by a diagonal argument.

Acknowledgment. I am grateful to Prof. Camillo De Lellis for some useful suggestions. Part of this research was done during a visit at the Laboratoire J. L. Lions of the University Paris VI in the framework of the RTN-Programme Fronts-Singularities. I am indebted to Prof. Haim Brezis for the invitation and for many stimulating discussions.

\section{Notation and preliminaries}

For a $p \times q$ matrix $A$ with $i j$-th entry $a_{i j}$, we denote by $|A|=\left(\Sigma_{i=1}^{p} \Sigma_{j=1}^{q} a_{i j}^{2}\right)^{1 / 2}$ the Frobenius norm of $A$.

For the $p \times q$ matrix $A$ with $i j$-th entry $a_{i j}$ and for the $q \times d$ matrix $B$ with $i j$-th entry $b_{i j}$ we denote by $A B:=A \cdot B$ their product, i.e., the $p \times d$ matrix, with $i j$-th entry $\sum_{k=1}^{q} a_{i k} b_{k j}$.

We identify $\boldsymbol{u}=\left(u_{1}, \ldots, u_{q}\right) \in \mathbb{R}^{q}$ with the $q \times 1$ matrix $A$ with $i 1$-st entry $u_{i}$, so that for the $p \times q$ matrix $A$ with $i j$-th entry $a_{i j}$ and for $\boldsymbol{v}=\left(v_{1}, v_{2}, \ldots, v_{q}\right) \in \mathbb{R}^{q}$ we denote by $A \boldsymbol{v}:=A \cdot \boldsymbol{v}$ the $p$-dimensional vector $\boldsymbol{u}=\left(u_{1}, \ldots, u_{p}\right) \in \mathbb{R}^{p}$, given by $u_{i}=\sum_{k=1}^{q} a_{i k} v_{k}$ for every $1 \leq i \leq p$.

As usual, $A^{T}$ denotes the transpose of the matrix $A$.

For $\boldsymbol{u}=\left(u_{1}, \ldots, u_{p}\right) \in \mathbb{R}^{p}$ and $\boldsymbol{v}=\left(v_{1}, \ldots, v_{p}\right) \in \mathbb{R}^{p}$, we denote by $\boldsymbol{u} \boldsymbol{v}:=$ $\boldsymbol{u} \cdot \boldsymbol{v}:=\sum_{k=1}^{p} u_{k} v_{k}$ the standard scalar product. Note that $\boldsymbol{u} \boldsymbol{v}=\boldsymbol{u}^{T} \boldsymbol{v}=\boldsymbol{v}^{T} \boldsymbol{u}$ as products of matrices. 
For $\boldsymbol{u}=\left(u_{1}, \ldots, u_{p}\right) \in \mathbb{R}^{p}$ and $\boldsymbol{v}=\left(v_{1}, \ldots, v_{q}\right) \in \mathbb{R}^{q}$, we denote by $\boldsymbol{u} \otimes \boldsymbol{v}$ the $p \times q$ matrix with $i j$-th entry $u_{i} v_{j}$ (i.e., $\boldsymbol{u} \otimes \boldsymbol{v}=\boldsymbol{u} \boldsymbol{v}^{T}$ as product of matrices).

For any $p \times q$ matrix $A$ with $i j$-th entry $a_{i j}$ and $\boldsymbol{v}=\left(v_{1}, v_{2}, \ldots, v_{d}\right) \in \mathbb{R}^{d}$, we denote by $A \otimes \boldsymbol{v}$ the $p \times q \times d$ tensor with $i j k$-th entry $a_{i j} v_{k}$.

Given a vector-valued function $f(x)=\left(f_{1}(x), \ldots, f_{k}(x)\right): \Omega \rightarrow \mathbb{R}^{k}\left(\Omega \subset \mathbb{R}^{N}\right)$, we denote by $D f$ or by $\nabla_{x} f$ the $k \times N$ matrix with $i j$-th entry $\frac{\partial f_{i}}{\partial x_{j}}$.

Given a matrix-valued function $F(x)=\left\{f_{i j}(x)\right\}(1 \leq i \leq p, 1 \leq j \leq q): \Omega \rightarrow \mathbb{R}^{p \times q}$, we denote by $D F$ or by $\nabla_{x} F$ the $p \times q \times N$ tensor with $i j k$-th entry $\frac{\partial f_{i j}}{\partial x_{k}}$.

For every dimension $d$, we denote by $I$ the unit $d \times d$-matrix and by $O$ the null $d \times d$-matrix.

Given a vector-valued measure $\mu=\left(\mu_{1}, \ldots, \mu_{k}\right)$ (where for any $1 \leq j \leq k, \mu_{j}$ is a finite signed measure), we denote by $\|\mu\|(E)$ its total variation measure of the set $E$.

For any $\mu$-measurable function $f$, we define the product measure $f \cdot \mu$ by $f \cdot \mu(E)=\int_{E} f d \mu$, for every $\mu$-measurable set $E$.

Throughout this paper, we assume that $\Omega \subset \mathbb{R}^{N}$ is a bounded Lipschitz domain.

In what follows, we present some known results on BV-spaces. We rely mainly on the book [3] by Ambrosio, Fusco and Pallara. Other sources are the books by Hudjaev and Volpert [25], Giusti [16] and Evans and Gariepy [13]. We begin by introducing some notation. For every $\nu \in S^{N-1}$ (the unit sphere in $\mathbb{R}^{N}$ ) and $R>0$, we set

$$
\begin{aligned}
& B_{R}^{+}(x, \boldsymbol{\nu})=\left\{y \in \mathbb{R}^{N}:|y-x|<R,(y-x) \cdot \boldsymbol{\nu}>0\right\}, \\
& B_{R}^{-}(x, \boldsymbol{\nu})=\left\{y \in \mathbb{R}^{N}:|y-x|<R,(y-x) \cdot \boldsymbol{\nu}<0\right\}, \\
& H_{+}(x, \boldsymbol{\nu})=\left\{y \in \mathbb{R}^{N}:(y-x) \cdot \boldsymbol{\nu}>0\right\}, \\
& H_{-}(x, \boldsymbol{\nu})=\left\{y \in \mathbb{R}^{N}:(y-x) \cdot \boldsymbol{\nu}<0\right\}
\end{aligned}
$$

and

$$
H_{\boldsymbol{\nu}}^{0}=\left\{y \in \mathbb{R}^{N}: y \cdot \boldsymbol{\nu}=0\right\} .
$$

Next we recall the definition of the space of functions with bounded variation. In what follows, $\mathcal{L}^{N}$ denotes Lebesgue measure in $\mathbb{R}^{N}$.

Definition 2.1. Let $\Omega$ be a domain in $\mathbb{R}^{N}$ and let $f \in L^{1}\left(\Omega, \mathbb{R}^{m}\right)$. We say that $f \in B V\left(\Omega, \mathbb{R}^{m}\right)$ if

$\int_{\Omega}|D f|:=\sup \left\{\int_{\Omega} \sum_{k=1}^{m} f_{k} \operatorname{div} \varphi_{k} d \mathcal{L}^{N}: \varphi_{k} \in C_{c}^{1}\left(\Omega, \mathbb{R}^{N}\right) ; \sum_{k=1}^{m}\left|\varphi_{k}(x)\right|^{2} \leq 1, x \in \Omega\right\}$

is finite. In this case, we define the BV-norm of $f$ by $\|f\|_{B V}:=\|f\|_{L^{1}}+\int_{\Omega}|D f|$. 
We recall below some basic notions in Geometric Measure Theory (see [3]).

Definition 2.2. Let $\Omega$ be a domain in $\mathbb{R}^{N}$. Consider a function $f \in L_{l o c}^{1}\left(\Omega, \mathbb{R}^{m}\right)$ and a point $x \in \Omega$.

i) We say that $x$ is a point of approximate continuity of $f$ if there exists $z \in \mathbb{R}^{m}$ such that

$$
\lim _{\rho \rightarrow 0^{+}} \frac{\int_{B_{\rho}(x)}|f(y)-z| d y}{\mathcal{L}^{N}\left(B_{\rho}(x)\right)}=0 .
$$

In this case $z$ is called an approximate limit of $f$ at $x$ and we write $z=\tilde{f}(x)$. The set of points of approximate continuity of $f$ is denoted by $G_{f}$.

ii) We say that $x$ is an approximate jump point of $f$ if there exist $a, b \in \mathbb{R}^{m}$ and $\nu \in S^{N-1}$ such that $a \neq b$ and

$$
\lim _{\rho \rightarrow 0^{+}} \frac{\int_{B_{\rho}^{+}(x, \boldsymbol{\nu})}|f(y)-a| d y}{\mathcal{L}^{N}\left(B_{\rho}(x)\right)}=0, \quad \lim _{\rho \rightarrow 0^{+}} \frac{\int_{B_{\rho}^{-}(x, \boldsymbol{\nu})}|f(y)-b| d y}{\mathcal{L}^{N}\left(B_{\rho}(x)\right)}=0 .
$$

The triple $(a, b, \nu)$, uniquely determined by (2.6) up to a permutation of $(a, b)$ and a change of sign of $\boldsymbol{\nu}$, is denoted by $\left(f^{+}(x), f^{-}(x), \boldsymbol{\nu}_{f}(x)\right)$. We call $\boldsymbol{\nu}_{f}(x)$ the approximate jump vector and sometimes write simply $\boldsymbol{\nu}(x)$ if the reference to the function $f$ is clear. The set of approximate jump points is denoted by $J_{f}$. A choice of $\boldsymbol{\nu}(x)$ for every $x \in J_{f}$ (which is unique up to sign) determines an orientation of $J_{f}$. At a point of approximate continuity $x$, we use the convention $f^{+}(x)=f^{-}(x)=\tilde{f}(x)$.

We recall the following results on $\mathrm{BV}$-functions that we use in the sequel. They are all taken from [3]. In all of them, $\Omega$ is a domain in $\mathbb{R}^{N}$ and $f$ belongs to $B V\left(\Omega, \mathbb{R}^{m}\right)$.

Theorem 2.1 (Theorems 3.69 and 3.78 from [3]).

i) $\mathcal{H}^{N-1}$-almost every point in $\Omega \backslash J_{f}$ is a point of approximate continuity of $f$.

ii) The set $J_{f}$ is a countably $\mathcal{H}^{N-1}$-rectifiable Borel set, oriented by $\boldsymbol{\nu}(x)$. In other words, $J_{f}$ is $\sigma$-finite with respect to $\mathcal{H}^{N-1}$, there exist countably many $C^{1}$ hypersurfaces $\left\{S_{k}\right\}_{k=1}^{\infty}$ such that $\mathcal{H}^{N-1}\left(J_{f} \backslash \bigcup_{k=1}^{\infty} S_{k}\right)=0$, and for $\mathcal{H}^{N-1}$ almost every $x \in J_{f} \cap S_{k}$, the approximate jump vector $\nu(x)$ is normal to $S_{k}$ at the point $x$.

iii) $\left[\left(f^{+}-f^{-}\right) \otimes \nu_{f}\right](x) \in L^{1}\left(J_{f}, d \mathcal{H}^{N-1}\right)$.

Theorem 2.2 (Theorems 3.92 and 3.78 from [3]). The distributional gradient $D f$ can be decomposed as a sum of three Borel regular finite matrix-valued measures on $\Omega$,

$$
D f=D^{a} f+D^{c} f+D^{j} f
$$


with

$$
D^{a} f=(\nabla f) \mathcal{L}^{N} \quad \text { and } \quad D^{j} f=\left(f^{+}-f^{-}\right) \otimes \nu_{f} \mathcal{H}^{N-1}\left\llcorner J_{f}\right.
$$

$D^{a}, D^{c}$ and $D^{j}$ are called absolutely continuous part, Cantor and jump part of $D f$, respectively, and $\nabla f \in L^{1}\left(\Omega, \mathbb{R}^{m \times N}\right)$ is the approximate differential of $f$. The three parts are mutually singular to each other. Moreover,

i) the support of $D^{c} f$ is concentrated on a set of $\mathcal{L}^{N}$-measure zero, but $\left(D^{c} f\right)(B)=0$ for any Borel set $B \subset \Omega$ which is $\sigma$-finite with respect to $\mathcal{H}^{N-1}$;

ii) $\left[D^{a} f\right]\left(f^{-1}(H)\right)=0$ and $\left[D^{c} f\right]\left(\tilde{f}^{-1}(H)\right)=0$ for every $H \subset \mathbb{R}^{m}$ satisfying $\mathcal{H}^{1}(H)=0$.

Theorem 2.3 (Volpert chain rule, Theorems 3.96 and 3.99 from [3]). Let $\Phi \in C^{1}\left(\mathbb{R}^{m}, \mathbb{R}^{q}\right)$ be a Lipschitz function satisfying $\Phi(0)=0$ if $|\Omega|=\infty$. Then, $v(x)=(\Phi \circ f)(x)$ belongs to $B V\left(\Omega, \mathbb{R}^{q}\right)$ and

$$
\begin{gathered}
D^{a} v=\nabla \Phi(f) \nabla f \mathcal{L}^{N}, D^{c} v=\nabla \Phi(\tilde{f}) D^{c} f, \\
D^{j} v=\left[\Phi\left(f^{+}\right)-\Phi\left(f^{-}\right)\right] \otimes \boldsymbol{\nu}_{f} \mathcal{H}^{N-1}\left\llcorner J_{f} .\right.
\end{gathered}
$$

We also recall that the trace operator $T$ is a continuous map from $B V(\Omega)$, endowed with the strong topology (or more generally, the topology induced by strict convergence), to $L^{1}\left(\partial \Omega, \mathcal{H}^{N-1}\llcorner\partial \Omega)\right.$, provided that $\Omega$ has a bounded Lipschitz boundary (see [3, Theorems 3.87 and 3.88]).

\section{First estimates on mollification}

Consider a function $\varphi=\left(\varphi_{1}, \varphi_{2}, \ldots, \varphi_{d}\right) \in B V\left(\mathbb{R}^{N}, \mathbb{R}^{d}\right)$ with compact support. Consider also a matrix-valued function $\eta \in C_{c}^{2}\left(\mathbb{R}^{N} \times \mathbb{R}^{N}, \mathbb{R}^{l \times d}\right)$. For any $\varepsilon>0$, define the function $\psi_{\varepsilon}(x): \mathbb{R}^{N} \rightarrow \mathbb{R}^{l}$ by

$\psi_{\varepsilon}(x):=\frac{1}{\varepsilon^{N}} \int_{\mathbb{R}^{N}} \eta\left(\frac{y-x}{\varepsilon}, x\right) \cdot \varphi(y) d y=\int_{\mathbb{R}^{N}} \eta(z, x) \cdot \varphi(x+\varepsilon z) d z, \quad$ for $x \in \mathbb{R}^{N}$.

The following technical lemma is used later in the upper bound construction.

\section{Lemma 3.1.}

$$
\int_{\mathbb{R}^{N}}\left|\psi_{\varepsilon}(x)-\left\{\int_{\mathbb{R}^{N}} \eta(z, x) d z\right\} \cdot \varphi(x)\right| d x=O(\varepsilon) \quad \text { as } \varepsilon \rightarrow 0 .
$$


Proof. By the definition of $\psi_{\varepsilon}$, we have

$$
\begin{aligned}
\int_{\mathbb{R}^{N}} \mid \psi_{\varepsilon}(x)- & \left\{\int_{\mathbb{R}^{N}} \eta(z, x) d z\right\} \cdot \varphi(x) \mid d x \\
& \leq \int_{\mathbb{R}^{N}} \int_{\mathbb{R}^{N}}|\eta(z, x)| \cdot|\varphi(x+\varepsilon z)-\varphi(x)| d z d x \\
& \leq \int_{B_{R}(0)}\|\eta\|_{L^{\infty}}\left(\int_{\mathbb{R}^{N}}|\varphi(x+\varepsilon z)-\varphi(x)| d x\right) d z
\end{aligned}
$$

where $R$ is a positive constant. Since

$$
\int_{\mathbb{R}^{N}}|\varphi(x+\varepsilon z)-\varphi(x)| d x \leq \varepsilon|z| \cdot\|D \varphi\|\left(\mathbb{R}^{N}\right)
$$

(see for example [3, Exercise 3.3]), we obtain from (3.2) that

$$
\int_{\mathbb{R}^{N}}\left|\psi_{\varepsilon}(x)-\left\{\int_{\mathbb{R}^{N}} \eta(z, x) d z\right\} \varphi(x)\right| d x \leq C \varepsilon\|D \varphi\|\left(\mathbb{R}^{N}\right) \int_{B_{R}(0)}|z| d z=O(\varepsilon) .
$$

Proposition 3.1. Let $W: \mathbb{R}^{l} \times \mathbb{R}^{q} \rightarrow \mathbb{R}$ be a $C^{1}$-function satisfying

$$
\nabla_{a} W(a, b)=0 \quad \text { whenever } W(a, b)=0 .
$$

Consider $\varphi \in B V\left(\mathbb{R}^{N}, \mathbb{R}^{d}\right) \cap L^{\infty}$ with compact support and $u \in B V\left(\mathbb{R}^{N}, \mathbb{R}^{q}\right) \cap L^{\infty}$ satisfying

$$
W\left(\left\{\int_{\mathbb{R}^{N}} \eta(z, x) d z\right\} \cdot \varphi(x), u(x)\right)=0 \quad \text { for a.e. } x \in \mathbb{R}^{N},
$$

where $\eta \in C_{c}^{2}\left(\mathbb{R}^{N} \times \mathbb{R}^{N}, \mathbb{R}^{l \times d}\right)$, as above, and let $\psi_{\varepsilon}(x)$ be as in (3.1). Then

$$
\begin{aligned}
& \lim _{\varepsilon \rightarrow 0} \int_{\mathbb{R}^{N}} \frac{1}{\varepsilon} W\left(\psi_{\varepsilon}(x), u(x)\right) d x \\
& =\int_{J_{\varphi}}\left\{\int_{-\infty}^{0} W\left(\gamma(t, x), u^{+}(x)\right) d t+\int_{0}^{\infty} W\left(\gamma(t, x), u^{-}(x)\right) d t\right\} d \mathcal{H}^{N-1}(x),
\end{aligned}
$$

where

$$
\gamma(t, x)=\left(\int_{-\infty}^{t} p(s, x) d s\right) \cdot \varphi^{-}(x)+\left(\int_{t}^{\infty} p(s, x) d s\right) \cdot \varphi^{+}(x)
$$

and

$$
p(t, x)=\int_{H_{\boldsymbol{\nu}(x)}^{0}} \eta(t \boldsymbol{\nu}(x)+y, x) d \mathcal{H}^{N-1}(y) .
$$

Here we assume that the orientation of $J_{u}$ coincides with the orientation of $J_{\varphi}$ $\mathcal{H}^{N-1}$ a.e. on $J_{u} \cap J_{\varphi}$. 
Proof. We may assume without loss of generality that $u$ is Borel measurable on $\mathbb{R}^{N}$.

Step 1: We prove the useful identity

$$
\begin{aligned}
& \int_{\mathbb{R}^{N}} \frac{1}{\varepsilon} W\left(\psi_{\varepsilon}(x), u(x)\right) d x \\
&=\int_{0}^{1}\left(\int _ { \mathbb { R } ^ { N } } \left\{\int_{B_{R}(0)} \nabla_{a} W\left(\psi_{t \varepsilon}(x-\varepsilon t z), u(x-\varepsilon t z)\right)\right.\right. \\
&\quad \times \eta(z, x) \cdot \sigma(x) \cdot z d z\} d\|D \varphi\|(x)) d t+o_{\varepsilon}(1),
\end{aligned}
$$

where $\sigma(x): \mathbb{R}^{N} \rightarrow \mathbb{R}^{d \times N}$ is the Radon-Nikodym derivative of $[D \varphi]$ with respect to $\|D \varphi\|$ (i.e., $[D \varphi]=\sigma \cdot\|D \varphi\|$ and $|\sigma|=1\|D \varphi\|$-almost everywhere) and $R>0$ is chosen so that $\eta(z, x)=0$ for all $|z|>R$ and $x \in \mathbb{R}^{N}$.

We set $\psi_{\varepsilon}(x)=\left(\psi_{1, \varepsilon}(x), \ldots, \psi_{l, \varepsilon}(x)\right)$ and denote the $i j$-th entry of $\eta(z, x)$ by $\eta_{i j}(z, x)$. For any $i \in\{1, \ldots, l\}$, any $t \in(0,1]$ and any $x \in \mathbb{R}^{N}$, we have

$$
\begin{aligned}
& \frac{d}{d t}\left(\psi_{i, t \varepsilon}(x)\right)= \frac{d}{d t}\left(\frac{1}{t^{N} \varepsilon^{N}} \int_{\mathbb{R}^{N}} \sum_{k=1}^{d} \eta_{i k}\left(\frac{y-x}{t \varepsilon}, x\right) \varphi_{k}(y) d y\right) \\
&=-\frac{1}{t^{N+1} \varepsilon^{N}} \int_{\mathbb{R}^{N}} \sum_{k=1}^{d}\left\{\nabla_{z} \eta_{i k}\left(\frac{y-x}{t \varepsilon}, x\right) \frac{y-x}{t \varepsilon}\right. \\
&\left.+N \eta_{i k}\left(\frac{y-x}{t \varepsilon}, x\right)\right\} \varphi_{k}(y) d y \\
&=-\frac{1}{t^{N} \varepsilon^{N-1}} \int_{\mathbb{R}^{N}} \sum_{k=1}^{d} \operatorname{div}_{y}\left\{\eta_{i k}\left(\frac{y-x}{t \varepsilon}, x\right) \frac{y-x}{t \varepsilon}\right\} \varphi_{k}(y) d y \\
&=\frac{1}{t^{N} \varepsilon^{N-1}} \int_{\mathbb{R}^{N}} \sum_{k=1}^{d} \eta_{i k}\left(\frac{y-x}{t \varepsilon}, x\right)\left(d\left[D \varphi_{k}\right](y)\right) \frac{y-x}{t \varepsilon},
\end{aligned}
$$

where $\nabla_{z} \eta_{i k}(z, x)$ denotes the partial gradient of $\eta_{i k}$ in the first variable. Therefore,

$$
\begin{aligned}
\frac{d}{d t} W\left(\psi_{t \varepsilon}(x), u(x)\right)= & \sum_{j=1}^{l} \frac{\partial W}{\partial a_{j}}\left(\psi_{t \varepsilon}(x), u(x)\right) \cdot \frac{d}{d t}\left(\psi_{j, \varepsilon t}(x)\right) \\
= & \varepsilon \nabla_{a} W\left(\psi_{t \varepsilon}(x), u(x)\right) \\
& \times\left[\frac{1}{t^{N} \varepsilon^{N}} \int_{\mathbb{R}^{N}} \eta\left(\frac{y-x}{t \varepsilon}, x\right) \cdot(d[D \varphi](y)) \cdot \frac{y-x}{t \varepsilon}\right]
\end{aligned}
$$


For any $\rho \in(0,1)$, we have by (3.9),

$$
\begin{aligned}
& \int_{\mathbb{R}^{N}} \frac{1}{\varepsilon}\left\{W\left(\psi_{\varepsilon}(x), u(x)\right)-W\left(\psi_{\rho \varepsilon}(x), u(x)\right)\right\} d x \\
& =\int_{\mathbb{R}^{N}} \frac{1}{\varepsilon}\left(\int_{\rho}^{1} \frac{d}{d t} W\left(\psi_{t \varepsilon}(x), u(x)\right)\right) d x \\
& =\int_{\mathbb{R}^{N}}\left\{\int_{\rho}^{1} \nabla_{a} W\left(\psi_{t \varepsilon}(x), u(x)\right)\left[\frac{1}{t^{N} \varepsilon^{N}} \int_{\mathbb{R}^{N}} \eta\left(\frac{y-x}{t \varepsilon}, x\right)(d[D \varphi](y)) \frac{y-x}{t \varepsilon}\right] d t\right\} d x \\
& =\int_{\rho}^{1}\left\{\int_{\mathbb{R}^{N}} \nabla_{a} W\left(\psi_{t \varepsilon}(x), u(x)\right)\left[\frac{1}{t^{N} \varepsilon^{N}} \int_{\mathbb{R}^{N}} \eta\left(\frac{y-x}{t \varepsilon}, x\right)(d[D \varphi](y)) \frac{y-x}{t \varepsilon}\right] d x\right\} d t \\
& =\int_{\rho}^{1}\left\{\int _ { \mathbb { R } ^ { N } } \left(\frac{1}{t^{N} \varepsilon^{N}} \int_{B_{R t \varepsilon}(y)} \nabla_{a} W\left(\psi_{t \varepsilon}(x), u(x)\right)\right.\right. \\
& \left.\left.\times \eta\left(\frac{y-x}{t \varepsilon}, x\right) \cdot \sigma(y) \cdot \frac{y-x}{t \varepsilon} d x\right) d\|D \varphi\|(y)\right\} d t .
\end{aligned}
$$

In this formulas as before, $\sigma(x): \mathbb{R}^{N} \rightarrow \mathbb{R}^{d \times N}$ is the Radon-Nikodym derivative of $[D \varphi]$ with respect to $\|D \varphi\|$ and $R>0$ is chosen so that $\eta(z, x)=0$ for all $|z|>R$ and $x \in \mathbb{R}^{N}$. From our assumptions on $W$, it follows that there exists a constant $C>0$, independent of $\rho$, such that $\left|\nabla_{a} W\left(\psi_{\rho}(x), u(x)\right)\right| \leq C$ for every $\rho>0$. Therefore, letting $\rho$ tend to zero in (3.10) and using Lemma 3.1, we get

$$
\begin{aligned}
\int_{\mathbb{R}^{N}} & \frac{1}{\varepsilon} W\left(\psi_{\varepsilon}(x), u(x)\right) d x \\
= & \int_{0}^{1}\left\{\int _ { \mathbb { R } ^ { N } } \left(\frac{1}{(t \varepsilon)^{N}} \int_{B_{R t \varepsilon}(y)} \nabla_{a} W\left(\psi_{t \varepsilon}(x), u(x)\right) \cdot \eta\left(\frac{y-x}{t \varepsilon}, x\right) \cdot \sigma(y)\right.\right. \\
\times & \left.\left.\frac{y-x}{t \varepsilon} d x\right) d\|D \varphi\|(y)\right\} d t \\
= & \int_{0}^{1}\left(\int _ { \mathbb { R } ^ { N } } \left\{\int_{B_{R}(0)} \nabla_{a} W\left(\psi_{t \varepsilon}(y-\varepsilon t z), u(y-\varepsilon t z)\right) \cdot \eta(z, y-\varepsilon t z)\right.\right. \\
& \times \sigma(y) \cdot z d z\} d\|D \varphi\|(y)) d t \\
& \int_{0}^{1}\left(\int _ { \mathbb { R } ^ { N } } \left\{\int_{B_{R}(0)} \begin{array}{r}
\nabla_{a} W\left(\psi_{t \varepsilon}(y-\varepsilon t z), u(y-\varepsilon t z)\right) \cdot \eta(z, y) \\
\times \sigma(y) \cdot z d z\} d\|D \varphi\|(y)) d t+o_{\varepsilon}(1)
\end{array}\right.\right.
\end{aligned}
$$

where in the last equality we have used the estimate $|\eta(z, y-\varepsilon t z)-\eta(z, y)| \leq C \varepsilon t|z|$. This proves identity in (3.7). 
Step 2: We prove that

$$
\begin{aligned}
& \text { (3.11) } \int_{\mathbb{R}^{N}} \frac{1}{\varepsilon} W\left(\psi_{\varepsilon}(x), u(x)\right) d x=o_{\varepsilon}(1)+ \\
& \int_{0}^{1}\left(\int_{J_{\varphi}}\left\{\int_{B_{R}(0)} \nabla_{a} W\left(\psi_{t \varepsilon}(x-\varepsilon t z), u(x-\varepsilon t z)\right) \cdot \eta(z, x) \cdot \sigma(x) \cdot z d z\right\} d\|D \varphi\|(x)\right) d t .
\end{aligned}
$$

Recall that $G_{\varphi} \subset \Omega$ is the set of approximate continuity of $\varphi$. For every $x$ in $G_{\varphi}$, we have

$$
\lim _{\rho \rightarrow 0^{+}} \frac{1}{\rho^{N}} \int_{B_{\rho}(x)}|\varphi(y)-\tilde{\varphi}(x)| d y=0 .
$$

Taking $\rho=2 R \varepsilon$ gives

$$
\lim _{\varepsilon \rightarrow 0^{+}} \int_{B_{2 R}(0)}|\varphi(x+\varepsilon z)-\tilde{\varphi}(x)| d z=0, \quad \text { for } x \text { in } G_{\varphi} .
$$

Since $\mathcal{H}^{N-1}\left(\mathbb{R}^{N} \backslash\left(G_{\varphi} \cup J_{\varphi}\right)\right)=0$ (see Theorem 2.1, i)) and the measure $[D \varphi]$ does not charge sets of $\mathcal{H}^{N-1}$-measure zero, we infer from (3.7) that

$$
\begin{aligned}
\int_{\mathbb{R}^{N}} & \left\{\int_{B_{R}(0)} \nabla_{a} W\left(\psi_{t \varepsilon}(x-\varepsilon t z), u(x-\varepsilon t z)\right) \cdot \eta(z, x) \cdot \sigma(x) \cdot z d z\right\} d\|D \varphi\|(x) \\
= & \int_{J_{\varphi}}\left\{\int_{B_{R}(0)} \nabla_{a} W\left(\psi_{t \varepsilon}(x-\varepsilon t z), u(x-\varepsilon t z)\right) \cdot \eta(z, x) \cdot \sigma(x) \cdot z d z\right\} d\|D \varphi\|(x) \\
& +\int_{G_{\varphi}}\left\{\int_{B_{R}(0)} \nabla_{a} W\left(\psi_{t \varepsilon}(x-\varepsilon t z), u(x-\varepsilon t z)\right) \cdot \eta(z, x) \cdot \sigma(x) \cdot z d z\right\} d\|D \varphi\|(x) .
\end{aligned}
$$

By (3.3), we have

$$
\nabla_{a} W\left(\left\{\int_{\mathbb{R}^{N}} \eta(z, x) d z\right\} \cdot \tilde{\varphi}(x), \tilde{u}(x)\right)=0 \quad \text { for all } x \in G_{\varphi} \cap G_{u} .
$$

Moreover, the set $G_{\varphi} \backslash G_{u}$ is $\mathcal{H}^{N-1} \sigma$-finite; therefore, by Theorem 2.2, we have $\|D \varphi\|\left(G_{\varphi} \backslash G_{u}\right)=0$. So

$$
\nabla_{a} W\left(\left\{\int_{\mathbb{R}^{N}} \eta(z, x) d z\right\} \cdot \tilde{\varphi}(x), \tilde{u}(x)\right)=0 \quad \text { for }\|D \varphi\| \text {-almost every } x \in G_{\varphi} .
$$

Therefore, by (3.12) and (3.14), the last integral in (3.13) tends to 0 when $\varepsilon \rightarrow 0$, for any $t \in(0,1]$. So by (3.7) and (3.13), we get (3.11).

Step 3: We claim that

$$
\lim _{\rho \rightarrow 0+} \psi_{\rho}(x-\rho z)=\gamma(\boldsymbol{\nu}(x) \cdot z, x) \quad \text { for all } x \in J_{\varphi}, z \in B_{R}(0) .
$$


For any $\rho \in(0,1), x \in J_{\varphi}$ and $z \in B_{R}(0)$, we have

$$
\begin{aligned}
\psi_{\rho}(x-\rho z)= & \int_{\mathbb{R}^{N}} \eta(y, x-\rho z) \cdot \varphi(x+\rho(y-z)) d y \\
= & \int_{B_{2 R}(0)} \eta(y+z, x-\rho z) \cdot \varphi(x+\rho y) d y \\
= & \int_{B_{2 R}^{+}(0, \boldsymbol{\nu}(x))} \eta(y+z, x-\rho z) \cdot \varphi(x+\rho y) d y \\
& +\int_{B_{2 R}^{-}(0, \boldsymbol{\nu}(x))} \eta(y+z, x-\rho z) \cdot \varphi(x+\rho y) d y
\end{aligned}
$$

By the definition of $J_{\varphi}$ (see (2.6)), for all $x \in J_{\varphi}$, we have

$\lim _{\varepsilon \rightarrow 0^{+}}\left\{\int_{B_{2 R}^{+}(0, \boldsymbol{\nu}(x))}\left|\varphi(x+\varepsilon z)-\varphi^{+}(x)\right| d z+\int_{B_{2 R}^{-}(0, \boldsymbol{\nu}(x))}\left|\varphi(x+\varepsilon z)-\varphi^{-}(x)\right| d z\right\}=0$.

Then, using (3.17) we infer that for every $x \in J_{\varphi}$ and every $z \in B_{R}(0)$, we have

$$
\begin{gathered}
\lim _{\rho \rightarrow 0^{+}} \int_{B_{2 R}^{+}(0, \boldsymbol{\nu}(x))} \eta(y+z, x-\rho z) \cdot \varphi(x+\rho y) d y \\
=\left(\int_{B_{2 R}^{+}(0, \boldsymbol{\nu}(x))} \eta(y+z, x) d y\right) \cdot \varphi^{+}(x), \\
\lim _{\rho \rightarrow 0^{+}} \int_{B_{2 R}^{-}(0, \boldsymbol{\nu}(x))} \eta(y+z, x-\rho z) \cdot \varphi(x+\rho y) d y \\
=\left(\int_{B_{2 R}^{-}(0, \boldsymbol{\nu}(x))} \eta(y+z, x) d y\right) \cdot \varphi^{-}(x) .
\end{gathered}
$$

\section{Note that}

$$
\begin{aligned}
& \int_{B_{2 R}^{+}(0, \boldsymbol{\nu}(x))} \eta(y+z, x) d y=\int_{H_{+}(0, \boldsymbol{\nu}(x))} \eta(y+z, x) d y=\int_{H_{+}(z, \boldsymbol{\nu}(x))} \eta(y, x) d y \\
= & \int_{\boldsymbol{\nu}(x) \cdot z}^{\infty}\left(\int_{H_{\boldsymbol{\nu}(x)}^{0}} \eta(t \boldsymbol{\nu}(x)+y, x) d \mathcal{H}^{N-1}(y)\right) d t=\int_{\boldsymbol{\nu}(x) \cdot z}^{\infty} p(t, x) d t \quad(\operatorname{see}(3.6)) .
\end{aligned}
$$

Similarly, we obtain

$$
\int_{B_{2 R}^{-}(0, \boldsymbol{\nu}(x))} \eta(y+z, x) d y=\int_{-\infty}^{\boldsymbol{\nu}(x) \cdot z} p(t, x) d t .
$$

Combining (3.16), (3.18), (3.19) and (3.20) we get that for every $x \in J_{\varphi}$ and every $z \in B_{R}(0)$ we have (see (3.5))

$$
\lim _{\rho \rightarrow 0+} \psi_{\rho}(x-\rho z)=\left(\int_{\boldsymbol{\nu}(x) \cdot z}^{\infty} p(t, x) d t\right) \cdot \varphi^{+}(x)+\left(\int_{-\infty}^{\boldsymbol{\nu}(x) \cdot z} p(t, x) d t\right) \cdot \varphi^{-}(x),
$$


and (3.15) follows.

Step 4: Completing the proof. Using (3.15) in (3.11), we obtain

$$
\begin{aligned}
& \int_{\mathbb{R}^{N}} \frac{1}{\varepsilon} W\left(\psi_{\varepsilon}(x), u(x)\right) d x \\
&=o_{\varepsilon}(1)+\int_{0}^{1}\left(\int _ { J _ { \varphi } } \left\{\int_{B_{R}(0)} \nabla_{a} W(\gamma(\boldsymbol{\nu}(x) \cdot z, x), u(x-\varepsilon t z))\right.\right.\times \eta(z, x) \cdot \sigma(x) \cdot z d z\} d\|D \varphi\|(x)) d t \\
&=o_{\varepsilon}(1)+\int_{0}^{1}\left(\int _ { J _ { \varphi } } \left\{\int_{B_{R}^{+}(0, \boldsymbol{\nu}(x))} \nabla_{a} W(\gamma(\boldsymbol{\nu}(x) \cdot z, x), u(x-\varepsilon t z))\right.\right. \\
&+\int_{0}^{1}\left(\int _ { J _ { \varphi } } \left\{\int_{B_{R}^{-}(0, \boldsymbol{\nu}(x))} \nabla_{a} W(\gamma(\boldsymbol{\nu}(x) \cdot z, x), u(x-\varepsilon t z))\right.\right. \\
&\times \eta(z, x) \cdot \sigma(x) \cdot z d z\} d\|D \varphi\|(x)) d t
\end{aligned}
$$

By the analogue of (3.17) for $u$, we infer that

$$
\begin{aligned}
& \int_{\mathbb{R}^{N}} \frac{1}{\varepsilon} W\left(\psi_{\varepsilon}(x), u(x)\right) d x \\
&=O_{\varepsilon}(1)+\int_{0}^{1}\left(\int _ { J _ { \varphi } } \left\{\int_{B_{R}^{+}(0, \boldsymbol{\nu}(x))} \nabla_{a} W\left(\gamma(\boldsymbol{\nu}(x) \cdot z, x), u^{-}(x)\right)\right.\right. \\
&\times \eta(z, x) \cdot \sigma(x) \cdot z d z\} d\|D \varphi\|(x)) d t \\
&+\int_{0}^{1}\left(\int _ { J _ { \varphi } } \left\{\int_{B_{R}^{-}(0, \boldsymbol{\nu}(x))} \nabla_{a} W\left(\gamma(\boldsymbol{\nu}(x) \cdot z, x), u^{+}(x)\right)\right.\right. \\
&\quad \times \eta(z, x) \cdot \sigma(x) \cdot z d z\} d\|D \varphi\|(x)) d t
\end{aligned}
$$

Here we have used the assumption that the orientation of $J_{u}$ coincides with the orientation of $J_{\varphi} \mathcal{H}^{N-1}$ a.e. on $J_{u} \cap J_{\varphi}$. By Theorem 2.2, we have

$$
\begin{aligned}
& \|D \varphi\|\left\llcorner J_{\varphi}=\left|\varphi^{+}-\varphi^{-}\right| \mathcal{H}^{N-1}\left\llcorner J_{\varphi}\right.\right. \\
& \qquad(x)=\frac{\left(\varphi^{+}(x)-\varphi^{-}(x)\right)}{\left|\varphi^{+}(x)-\varphi^{-}(x)\right|} \otimes \nu(x) \quad \text { for } \mathcal{H}^{N-1} \text {-almost every } x \in J_{\varphi} .
\end{aligned}
$$


Then, by (3.21), we have

$$
\begin{aligned}
& \int_{\mathbb{R}^{N}} \frac{1}{\varepsilon} W\left(\psi_{\varepsilon}(x), u(x)\right) d x \\
&=o_{\varepsilon}(1)+\int_{J_{\varphi}}\left\{\int_{B_{R}^{+}(0, \boldsymbol{\nu}(x))} \nabla_{a} W\left(\gamma(\boldsymbol{\nu}(x) \cdot z, x), u^{-}(x)\right) \cdot \eta(z, x)\right. \\
&\left.\times\left(\left(\varphi^{+}(x)-\varphi^{-}(x)\right) \otimes \boldsymbol{\nu}(x)\right) \cdot z d z\right\} d \mathcal{H}^{N-1}(x) \\
&+\int_{J_{\varphi}}\left\{\int_{B_{R}^{-}(0, \boldsymbol{\nu}(x))} \nabla_{a} W\left(\gamma(\boldsymbol{\nu}(x) \cdot z, x), u^{+}(x)\right) \cdot \eta(z, x)\right. \\
&\left.\times\left(\left(\varphi^{+}(x)-\varphi^{-}(x)\right) \otimes \boldsymbol{\nu}(x)\right) \cdot z d z\right\} d \mathcal{H}^{N-1}(x)
\end{aligned}
$$

\section{Note that}

$$
\begin{aligned}
& \int_{B_{R}^{+}(0, \boldsymbol{\nu}(x))} \nabla_{a} W\left(\gamma(\boldsymbol{\nu}(x) \cdot z, x), u^{-}(x)\right) \cdot \eta(z, x) \cdot\left(\left(\varphi^{+}(x)-\varphi^{-}(x)\right) \otimes \boldsymbol{\nu}(x)\right) \cdot z d z \\
& =\int_{H_{+}(0, \boldsymbol{\nu}(x))} \nabla_{a} W\left(\gamma(\boldsymbol{\nu}(x) \cdot z, x), u^{-}(x)\right) \\
& \quad \times \eta(z, x) \cdot\left(\varphi^{+}(x)-\varphi^{-}(x)\right) \cdot(\boldsymbol{\nu}(x))^{T} \cdot z d z
\end{aligned}
$$

In the same way, we have

$$
\begin{array}{r}
\int_{B_{R}^{-}(0, \boldsymbol{\nu}(x))} \nabla_{a} W\left(\gamma(\boldsymbol{\nu}(x) \cdot z, x), u^{-}(x)\right) \cdot \eta(z, x) \cdot\left(\left(\varphi^{+}(x)-\varphi^{-}(x)\right) \otimes \boldsymbol{\nu}(x)\right) \cdot z d z \\
=\left(\int_{-\infty}^{0} \nabla_{a} W\left(\gamma(\tau, x), u^{+}(x)\right) \cdot p(\tau, x) \tau d \tau\right) \cdot\left(\varphi^{+}(x)-\varphi^{-}(x)\right)
\end{array}
$$


Therefore, by (3.22), (3.23), (3.24)

$$
\begin{aligned}
& \int_{\mathbb{R}^{N}} \frac{1}{\varepsilon} W\left(\psi_{\varepsilon}(x), u(x)\right) d x \\
=o_{\varepsilon}(1) & +\int_{J_{\varphi}}\left(\int_{0}^{\infty} \nabla_{a} W\left(\gamma(\tau, x), u^{-}(x)\right) \cdot p(\tau, x) \tau d \tau\right)\left(\varphi^{+}(x)-\varphi^{-}(x)\right) d \mathcal{H}^{N-1}(x) \\
& +\int_{J_{\varphi}}\left(\int_{-\infty}^{0} \nabla_{a} W\left(\gamma(\tau, x), u^{+}(x)\right) \cdot p(\tau, x) \tau d \tau\right)\left(\varphi^{+}(x)-\varphi^{-}(x)\right) d \mathcal{H}^{N-1}(x) .
\end{aligned}
$$

Next, since $\frac{\partial \gamma(\tau, x)}{\partial \tau}=-p(\tau, x) \cdot\left(\varphi^{+}(x)-\varphi^{-}(x)\right)$ for $\mathcal{H}^{N-1}$-a.e. $x \in J_{\varphi}$, we have

$$
\begin{aligned}
\int_{0}^{\infty} \nabla_{a} W\left(\gamma(\tau, x), u^{-}(x)\right) \cdot p(\tau, x) \cdot\left(\varphi^{+}(x)-\varphi^{-}(x)\right) \tau d \tau \\
=\int_{0}^{\infty} W\left(\gamma(\tau, x), u^{-}(x)\right) d \tau \\
\int_{-\infty}^{0} \nabla_{a} W\left(\gamma(\tau, x), u^{+}(x)\right) \cdot p(\tau, x) \cdot\left(\varphi^{+}(x)-\varphi^{-}(x)\right) \tau d \tau \\
=\int_{-\infty}^{0} W\left(\gamma(\tau, x), u^{+}(x)\right) d \tau .
\end{aligned}
$$

Plugging (3.26) in (3.25) gives the desired result (3.4).

Remark 3.1. For any $\varphi \in B V\left(\Omega, \mathbb{R}^{d}\right)$, we may by [3, Proposition 3.21] extend $\varphi$ to a function $\bar{\varphi} \in B V\left(\mathbb{R}^{N}, \mathbb{R}^{d}\right)$ such that $\bar{\varphi}=\varphi$ a.e. in $\Omega, \operatorname{supp} \bar{\varphi}$ is compact and $\|D \bar{\varphi}\|(\partial \Omega)=0$. From the proof of Proposition 3.21 in [3], it follows that if $\varphi \in B V\left(\Omega, \mathbb{R}^{d}\right) \cap L^{\infty}$, then its extension $\bar{\varphi}$ is also in $B V\left(\mathbb{R}^{N}, \mathbb{R}^{d}\right) \cap L^{\infty}$.

Consider a function $\varphi=\left(\varphi_{1}, \varphi_{2}, \ldots, \varphi_{d}\right) \in B V\left(\Omega, \mathbb{R}^{d}\right)$, together with its extension $\bar{\varphi}$ (as in Remark 3.1). Consider $\eta \in C_{c}^{2}\left(\mathbb{R}^{N} \times \mathbb{R}^{N}, \mathbb{R}^{l \times d}\right)$ and set

$\psi_{\varepsilon}(x):=\frac{1}{\varepsilon^{N}} \int_{\mathbb{R}^{N}} \eta\left(\frac{y-x}{\varepsilon}, x\right) \cdot \bar{\varphi}(y) d y=\int_{\mathbb{R}^{N}} \eta(z, x) \cdot \bar{\varphi}(x+\varepsilon z) d z, \quad$ for $x \in \mathbb{R}^{N}$.

We have the following analogue of Proposition 3.1 for bounded domains.

Proposition 3.2. Let $W \in C^{1}\left(\mathbb{R}^{l} \times \mathbb{R}^{q}, \mathbb{R}\right)$ satisfy

$$
\nabla_{a} W(a, b)=0 \text { whenever } W(a, b)=0 .
$$

Consider $\varphi \in B V\left(\Omega, \mathbb{R}^{d}\right) \cap L^{\infty}$ and $u \in B V\left(\Omega, \mathbb{R}^{q}\right) \cap L^{\infty}$ satisfying

$$
W\left(\left\{\int_{\mathbb{R}^{N}} \eta(z, x) d z\right\} \cdot \varphi(x), u(x)\right)=0 \quad \text { for a.e. } x \in \Omega,
$$


where $\eta \in C_{c}^{2}\left(\mathbb{R}^{N} \times \mathbb{R}^{N}, \mathbb{R}^{l \times d}\right)$. Let $\psi_{\varepsilon}(x)$ be as in (3.27). Then

$$
\begin{aligned}
& \lim _{\varepsilon \rightarrow 0} \int_{\Omega} \frac{1}{\varepsilon} W\left(\psi_{\varepsilon}(x), u(x)\right) d x \\
= & \int_{J_{\varphi}}\left\{\int_{-\infty}^{0} W\left(\gamma(t, x), u^{+}(x)\right) d t+\int_{0}^{\infty} W\left(\gamma(t, x), u^{-}(x)\right) d t\right\} d \mathcal{H}^{N-1}(x),
\end{aligned}
$$

where

$$
\gamma(t, x)=\left(\int_{-\infty}^{t} p(s, x) d s\right) \cdot \varphi^{-}(x)+\left(\int_{t}^{\infty} p(s, x) d s\right) \cdot \varphi^{+}(x),
$$

and

$$
p(t, x)=\int_{H_{\nu(x)}^{0}} \eta(t \boldsymbol{\nu}(x)+y, x) d \mathcal{H}^{N-1}(y) .
$$

Here we assume that the orientation of $J_{u}$ coincides with the orientation of $J_{\varphi}$ $\mathcal{H}^{N-1}$ a.e. on $J_{u} \cap J_{\varphi}$.

Proof. Consider some $B V$ extension $\bar{u}(x):=\left(\bar{u}_{1}(x), \ldots, \bar{u}_{q}(x)\right)$ of $u$ to $\mathbb{R}^{N}$. Then define $\bar{U}(x): \mathbb{R}^{N} \rightarrow \mathbb{R}^{q+1}$ by $\bar{U}(x):=\left(\bar{u}_{1}(x), \ldots, \bar{u}_{q}(x), \chi_{\Omega}(x)\right)$, where $\chi_{\Omega}$ is the characteristic function of $\Omega$. Then $\bar{U} \in B V\left(\mathbb{R}^{N}, \mathbb{R}^{q+1}\right)$. Next define $\bar{W}(a, b): \mathbb{R}^{l} \times \mathbb{R}^{q+1} \rightarrow \mathbb{R}$ by $\bar{W}(a, b):=b_{q+1}^{2} \cdot W\left(a, b^{\prime}\right)$, where $b=\left(b_{1}, \ldots, b_{q}, b_{q+1}\right)=$ $\left(b^{\prime}, b_{q+1}\right)$. Thus,

$$
\bar{W}\left(\psi_{\varepsilon}(x), \bar{U}(x)\right)=\left(\chi_{\Omega}(x)\right)^{2} \cdot W\left(\psi_{\varepsilon}(x), \bar{u}(x)\right) .
$$

Applying Proposition 3.1 with $\bar{\varphi}, \bar{U}$ and $\bar{W}$ in place of $\varphi, u$ and $W$, and using the fact that $\|D \bar{\varphi}\|(\partial \Omega)=0$, which gives $\mathcal{H}^{N-1}\left(J_{\bar{\varphi}} \cap \partial \Omega\right)=0$, we complete the proof.

\section{The upper bound construction}

We define a special class of mollifiers that we use in the upper bound construction. Note that in contrast with standard mollifiers, our mollifiers depend on two variables.

Definition 4.1. The class $\mathcal{V}$ consists of all functions $\eta \in C_{c}^{2}\left(\mathbb{R}^{N} \times \mathbb{R}^{N}, \mathbb{R}^{d \times d}\right)$ such that

$$
\int_{\mathbb{R}^{N}} \eta(z, x) d z=I \quad \text { for all } x \in \Omega \quad(I \text { is the identity matrix }) .
$$


For $\varphi \in B V\left(\Omega, \mathbb{R}^{d}\right) \cap L^{\infty}$ and $\eta \in \mathcal{V}$, let $R>0$ be such that $\eta(z, x)=0$ whenever $|z|>R$. As in Section 3, we may by [3, Proposition 3.21] extend $\varphi$ to a function $\bar{\varphi} \in B V\left(\mathbb{R}^{N}, \mathbb{R}^{d}\right) \cap L^{\infty}$ such that $\bar{\varphi}=\varphi$ a.e. in $\Omega$, $\operatorname{supp} \bar{\varphi}$ is compact and $\|D \bar{\varphi}\|(\partial \Omega)=0$ (see Remark 3.1). For every $\varepsilon>0$, define a function $\psi_{\varepsilon} \in C^{1}\left(\mathbb{R}^{N}, \mathbb{R}^{d}\right)$, as in (3.27), by

$$
\psi_{\varepsilon}(x):=\frac{1}{\varepsilon^{N}} \int_{\mathbb{R}^{N}} \eta\left(\frac{y-x}{\varepsilon}, x\right) \cdot \bar{\varphi}(y) d y=\int_{\mathbb{R}^{N}} \eta(z, x) \cdot \bar{\varphi}(x+\varepsilon z) d z .
$$

Then by Lemma 3.1, we have

$$
\int_{\Omega}\left|\psi_{\varepsilon}(x)-\varphi(x)\right| d x=O(\varepsilon) \quad \text { as } \varepsilon \rightarrow 0 .
$$

Theorem 4.1. Let $H \in C^{1}\left(\mathbb{R}^{d \times N} \times \mathbb{R}^{d} \times \mathbb{R}^{q}, \mathbb{R}\right)$ satisfy $H(a, b, c) \geq 0$ for every $(a, b, c)$. Consider $u \in B V\left(\Omega, \mathbb{R}^{q}\right) \cap L^{\infty}$ and $\varphi \in B V\left(\Omega, \mathbb{R}^{d}\right) \cap L^{\infty}$ satisfying

$$
H(O, \varphi(x), u(x))=0 \quad \text { for a.e. } x \in \Omega .
$$

For any $\eta(z, x):=\left\{\eta_{i j}(z, x)\right\} \in \mathcal{V}$, let $\psi_{\varepsilon}$ be defined by (4.2). Then

$$
\begin{aligned}
\lim _{\varepsilon \rightarrow 0} & \int_{\Omega} \frac{1}{\varepsilon} H\left(\varepsilon \nabla \psi_{\varepsilon}, \psi_{\varepsilon}, u\right) d x \\
= & \int_{J_{\varphi}}\left\{\int_{-\infty}^{0} H\left(p(t, x) \cdot\left(\varphi^{+}(x)-\varphi^{-}(x)\right) \otimes \boldsymbol{\nu}(x), \gamma(t, x), u^{+}(x)\right) d t\right. \\
& \left.+\int_{0}^{\infty} H\left(p(t, x) \cdot\left(\varphi^{+}(x)-\varphi^{-}(x)\right) \otimes \boldsymbol{\nu}(x), \gamma(t, x), u^{-}(x)\right) d t\right\} d \mathcal{H}^{N-1}(x),
\end{aligned}
$$

where $p$ and $\gamma$ are defined in (3.6) and (3.5), respectively, and it is assumed that the orientation of $J_{u}$ coincides with the orientation of $J_{\varphi} \mathcal{H}^{N-1}$ a.e. on $J_{u} \cap J_{\varphi}$.

Before proving Theorem 4.1, we state and prove the following important corollary.

Corollary 4.1. Let $H, u$ and $\varphi$ be as in Theorem 4.1. Then for every $\eta \in \mathcal{V}$, there exists a family of functions $\left\{\varphi_{\varepsilon}\right\} \subset C^{2}\left(\mathbb{R}^{N}, \mathbb{R}^{d \times d}\right)$ such that $\int_{\Omega} \varphi_{\varepsilon}(x) d x=$ $\int_{\Omega} \varphi(x) d x, \varphi_{\varepsilon}(x) \rightarrow \varphi(x)$ in $L^{p}\left(\Omega, \mathbb{R}^{d \times d}\right)$ for every $p \in[1, \infty)$ and

$$
\begin{aligned}
\lim _{\varepsilon \rightarrow 0} & \int_{\Omega} \frac{1}{\varepsilon} H\left(\varepsilon \nabla \varphi_{\varepsilon}, \varphi_{\varepsilon}, u\right) d x \\
= & \int_{J_{\varphi}}\left\{\int_{-\infty}^{0} H\left(p(t, x) \cdot\left(\varphi^{+}(x)-\varphi^{-}(x)\right) \otimes \boldsymbol{\nu}(x), \gamma(t, x), u^{+}(x)\right) d t\right. \\
& \left.\quad+\int_{0}^{\infty} H\left(p(t, x) \cdot\left(\varphi^{+}(x)-\varphi^{-}(x)\right) \otimes \boldsymbol{\nu}(x), \gamma(t, x), u^{-}(x)\right) d t\right\} d \mathcal{H}^{N-1}(x) .
\end{aligned}
$$


Proof of the Corollary. Let $\psi_{\varepsilon}$ be defined by (4.2). Then $\psi_{\varepsilon}(x) \rightarrow \varphi(x)$ in $L^{p}\left(\Omega, \mathbb{R}^{d \times d}\right)$; and by Theorem 4.1 , we have (4.4). Consider

$$
\begin{aligned}
d_{\varepsilon} & :=\int_{\Omega} \psi_{\varepsilon}(x) d x-\int_{\Omega} \varphi(x) d x=\int_{\Omega}\left(\int_{B_{R}(0)} \eta(z, x)(\bar{\varphi}(x+\varepsilon z)-\bar{\varphi}(x)) d z\right) d x \\
& =\int_{B_{R}(0)}\left(\int_{\Omega} \eta(z, x)(\bar{\varphi}(x+\varepsilon z)-\bar{\varphi}(x)) d x\right) d z .
\end{aligned}
$$

Since

$$
\int_{\Omega}|\bar{\varphi}(x+\varepsilon z)-\bar{\varphi}(x)| d x \leq \varepsilon|z| \cdot\|D \bar{\varphi}\|\left(\mathbb{R}^{N}\right),
$$

we obtain

$$
\left|d_{\varepsilon}\right| \leq \int_{B_{R}(0)}\left(\int_{\Omega}|\eta(z, x)||\bar{\varphi}(x+\varepsilon z)-\bar{\varphi}(x)| d x\right) d z \leq \varepsilon C\|D \bar{\varphi}\|\left(\mathbb{R}^{N}\right)=O(\varepsilon) .
$$

Consider $\varphi_{\varepsilon}(x):=\psi_{\varepsilon}(x)-d_{\varepsilon}$. Then $\int_{\Omega} \varphi_{\varepsilon}(x) d x=\int_{\Omega} \varphi(x) d x$, and $\varphi_{\varepsilon}(x) \rightarrow \varphi(x)$ in $L^{p}\left(\Omega, \mathbb{R}^{d \times d}\right)$. In order to prove (4.5), it is sufficient to show that

$$
\lim _{\varepsilon \rightarrow 0} \int_{\Omega} \frac{1}{\varepsilon}\left|H\left(\varepsilon \nabla \varphi_{\varepsilon}, \varphi_{\varepsilon}, u\right)-H\left(\varepsilon \nabla \psi_{\varepsilon}, \psi_{\varepsilon}, u\right)\right| d x=0
$$

We have

$$
\begin{aligned}
H\left(\varepsilon \nabla \varphi_{\varepsilon}, \varphi_{\varepsilon}, u\right)-H\left(\varepsilon \nabla \psi_{\varepsilon}, \psi_{\varepsilon}, u\right) & =H\left(\varepsilon \nabla \psi_{\varepsilon}, \psi_{\varepsilon}-d_{\varepsilon}, u\right)-H\left(\varepsilon \nabla \psi_{\varepsilon}, \psi_{\varepsilon}, u\right) \\
& =-\left(\int_{0}^{1} \nabla_{b} H\left(\varepsilon \nabla \psi_{\varepsilon}, \psi_{\varepsilon}-t d_{\varepsilon}, u\right) d t\right) \cdot d_{\varepsilon}
\end{aligned}
$$

Therefore, using (4.6), we infer

$$
\begin{aligned}
\int_{\Omega} \frac{1}{\varepsilon} \mid H\left(\varepsilon \nabla \varphi_{\varepsilon}, \varphi_{\varepsilon}, u\right)-H( & \left.\varepsilon \nabla \psi_{\varepsilon}, \psi_{\varepsilon}, u\right) \mid d x \\
& \leq C \int_{0}^{1} \int_{\Omega}\left|\nabla_{b} H\left(\varepsilon \nabla \psi_{\varepsilon}, \psi_{\varepsilon}-t d_{\varepsilon}, u\right)\right| d x d t
\end{aligned}
$$

On the other hand, for any $1 \leq j \leq N$, we have

$$
\begin{aligned}
\frac{\partial \psi_{\varepsilon}(x)}{\partial x_{j}} & =\frac{1}{\varepsilon^{N}} \int_{\mathbb{R}^{N}} \frac{\partial}{\partial x_{j}}\left\{\eta\left(\frac{y-x}{\varepsilon}, x\right)\right\} \cdot \bar{\varphi}(y) d y \\
& =\frac{1}{\varepsilon^{N}} \int_{\mathbb{R}^{N}}\left\{-\frac{1}{\varepsilon} \frac{\partial}{\partial \alpha_{j}} \eta\left(\frac{y-x}{\varepsilon}, x\right)+\frac{\partial}{\partial \beta_{j}} \eta\left(\frac{y-x}{\varepsilon}, x\right)\right\} \cdot \bar{\varphi}(y) d y \\
& =\int_{\mathbb{R}^{N}}\left\{-\frac{1}{\varepsilon} \frac{\partial}{\partial z_{j}} \eta(z, x)+\frac{\partial}{\partial x_{j}} \eta(z, x)\right\} \cdot \bar{\varphi}(x+\varepsilon z) d z
\end{aligned}
$$


where $\alpha=\left(\alpha_{1}, \ldots, \alpha_{N}\right)$ and $\beta=\left(\beta_{1}, \ldots, \beta_{N}\right)$ are the first and the second arguments of the function $\eta(\alpha, \beta): \mathbb{R}^{N} \times \mathbb{R}^{N} \rightarrow \mathbb{R}^{d \times d}$. Therefore,

$$
\begin{aligned}
\varepsilon \frac{\partial \psi_{\varepsilon}(x)}{\partial x_{j}}= & -\int_{\mathbb{R}^{N}} \frac{\partial \eta(z, x)}{\partial z_{j}} \cdot \bar{\varphi}(x+\varepsilon z) d z+\varepsilon \int_{\mathbb{R}^{N}} \frac{\partial \eta(z, x)}{\partial x_{j}} \cdot \bar{\varphi}(x+\varepsilon z) d z \\
= & -\int_{\mathbb{R}^{N}} \frac{\partial \eta(z, x)}{\partial z_{j}} \cdot(\bar{\varphi}(x+\varepsilon z)-\varphi(x)) d z \\
& +\varepsilon \int_{\mathbb{R}^{N}} \frac{\partial \eta(z, x)}{\partial x_{j}} \cdot(\bar{\varphi}(x+\varepsilon z)-\bar{\varphi}(x)) d z
\end{aligned}
$$

The last equality follows from $\int_{\mathbb{R}^{N}} \frac{\partial \eta(z, x)}{\partial x_{j}} d z=\int_{\mathbb{R}^{N}} \frac{\partial \eta(z, x)}{\partial z_{j}} d z=O$ (see (4.1)). In particular, $\varepsilon \nabla \psi_{\varepsilon}(x) \rightarrow 0$ for a.e. $x$. Since $\nabla_{b} H(O, \varphi(x), u(x))=0$ a.e. in $\Omega$ and $\nabla_{b} H$ is continuous and bounded, using (4.8) and (4.10), we infer (4.7).

Proof of Theorem 4.1. As before in (4.10), for any $1 \leq j \leq N$, we obtain

$$
\varepsilon \frac{\partial \psi_{\varepsilon}(x)}{\partial x_{j}}=-\int_{\mathbb{R}^{N}} \frac{\partial \eta(z, x)}{\partial z_{j}} \cdot \bar{\varphi}(x+\varepsilon z) d z+\varepsilon \int_{\mathbb{R}^{N}} \frac{\partial \eta(z, x)}{\partial x_{j}} \cdot(\bar{\varphi}(x+\varepsilon z)-\bar{\varphi}(x)) d z .
$$

Next let $M(z, x):=\left\{M_{i j}(z, x)\right\}: \mathbb{R}^{N} \times \mathbb{R}^{N} \rightarrow \mathbb{R}^{d(N+1) \times d}$ be defined by

$$
\left\{\begin{array}{l}
M_{i j}(z, x):=\eta_{i j}(z, x) \\
M_{(d k+i) j}(z, x):=-\frac{\partial}{\partial z_{k}} \eta_{i j}(z, x)
\end{array} \quad \text { for all } 1 \leq i \leq d, 1 \leq j \leq d, 1 \leq k \leq N\right.
$$

Then

$$
\left\{\begin{array}{l}
\int_{\mathbb{R}^{N}} M_{i j}(z, x) d z=\delta_{i j} \\
\int_{\mathbb{R}^{N}} M_{(d k+i) j}(z, x) d z=0
\end{array} \quad \text { for all } 1 \leq i \leq d, 1 \leq j \leq d, 1 \leq k \leq N\right.
$$

Set

$$
\Psi_{\varepsilon}(x):=\frac{1}{\varepsilon^{N}} \int_{\mathbb{R}^{N}} M\left(\frac{y-x}{\varepsilon}, x\right) \cdot \bar{\varphi}(y) d y=\int_{\mathbb{R}^{N}} M(z, x) \cdot \bar{\varphi}(x+\varepsilon z) d z .
$$

Finally, let the function $W(A, B): \mathbb{R}^{d(N+1)} \times \mathbb{R}^{q} \rightarrow \mathbb{R}$ be defined as follows. For $A=\left(A_{1}, \ldots, A_{d(N+1)}\right) \in \mathbb{R}^{d(N+1)}$ consider

- the $d \times N$-matrix $a$ with $i j$-th entry $A_{d j+i}$,

- $b:=\left(A_{1}, \ldots, A_{d}\right)$,

- $c=B$

and define

$$
W(A, B):=H(a, b, c) .
$$


Then $W \in C^{1}, W \geq 0$ and therefore $\nabla_{A} W(A, B)=0$ whenever $W(A, B)=0$. Using the fact that $H(O, \varphi(x), u(x))=0$ for a.e. $x \in \Omega$, we infer from (4.13) that

$$
W\left(\left\{\int_{\mathbb{R}^{N}} M(z, x) d z\right\} \cdot \varphi(x), u(x)\right)=0 \quad \text { for a.e. } x \in \Omega .
$$

Therefore, using Proposition 3.2, we conclude that

$$
\text { (4.14) } \begin{aligned}
& \lim _{\varepsilon \rightarrow 0} \int_{\Omega} \frac{1}{\varepsilon} W\left(\Psi_{\varepsilon}(x), u(x)\right) d x \\
= & \int_{J_{\varphi}}\left\{\int_{-\infty}^{0} W\left(\Gamma(t, x), u^{+}(x)\right) d t+\int_{0}^{\infty} W\left(\Gamma(t, x), u^{-}(x)\right) d t\right\} d \mathcal{H}^{N-1}(x),
\end{aligned}
$$

where

$$
\Gamma(t, x)=\left(\int_{-\infty}^{t} P(s, x) d s\right) \cdot \varphi^{-}(x)+\left(\int_{t}^{\infty} P(s, x) d s\right) \cdot \varphi^{+}(x),
$$

with

$$
P(t, x)=\left\{P_{i j}(t, x)\right\}:=\int_{H_{\boldsymbol{\nu}(x)}^{0}} M(t \boldsymbol{\nu}(x)+y, x) d \mathcal{H}^{N-1}(y),
$$

Let $\boldsymbol{e}_{1}=(1,0,0, \ldots, 0), \boldsymbol{e}_{2}=(0,1,0, \ldots, 0), \ldots, \boldsymbol{e}_{N}=(0, \ldots, 0,1)$, and let $\boldsymbol{\zeta}_{1}, \ldots, \boldsymbol{\zeta}_{N-1}$ be an arbitrary orthonormal base of $H_{\boldsymbol{\nu}(x)}^{0}$. Then, for any $1 \leq j \leq N$, we have

$$
\frac{\partial \eta(z, x)}{\partial z_{j}}=\frac{d_{z} \eta(z, x)}{d_{z} \boldsymbol{e}_{j}}=\nu_{j}(x) \frac{d_{z} \eta(z, x)}{d_{z} \boldsymbol{\nu}(x)}+\sum_{k=1}^{N-1}\left(\boldsymbol{\zeta}_{k} \cdot \boldsymbol{e}_{j}\right) \frac{d_{z} \eta(z, x)}{d_{z} \boldsymbol{\zeta}_{k}}
$$

where $\left(\nu_{1}(x), \ldots, \nu_{N}(x)\right)=\boldsymbol{\nu}(x)$. But for any $k$, we have

$$
\int_{H_{\boldsymbol{\nu}(x)}^{0}} \frac{d_{z}}{d_{z} \boldsymbol{\zeta}_{k}} \eta(t \boldsymbol{\nu}(x)+y, x) d \mathcal{H}^{N-1}(y)=0 .
$$

Therefore,

$$
\begin{aligned}
\int_{H_{\boldsymbol{\nu}(x)}^{0}} \frac{\partial}{\partial z_{j}} & \eta(t \boldsymbol{\nu}(x)+y, x) d \mathcal{H}^{N-1}(y) \\
& =\nu_{j}(x) \frac{d}{d t}\left\{\int_{H_{\boldsymbol{\nu}(x)}^{0}} \eta(t \boldsymbol{\nu}(x)+y, x) d \mathcal{H}^{N-1}(y)\right\} \\
& =\nu_{j}(x) \frac{d p(t, x)}{d t} .
\end{aligned}
$$

Plugging (4.17) and (4.12) into (4.16), we get (4.18)

$$
\left\{\begin{array}{l}
P_{i j}(t, x) d z=p_{i j}(t, x) \\
P_{(d k+i) j}(t, x)=-\nu_{k}(x) \frac{d p_{i j}(t, x)}{d t}
\end{array} \quad \text { for all } 1 \leq i \leq d, 1 \leq j \leq d, 1 \leq k \leq N .\right.
$$


Therefore, writing $\Gamma(t, x)=\left(\Gamma_{1}(t, x), \ldots, \Gamma_{d(N+1)}(t, x)\right)$, we have

$$
\left(\Gamma_{j d+1}, \ldots, \Gamma_{j d+d}(t, x)\right)=\nu_{j}(x) p(t, x) \cdot\left(\varphi^{+}(x)-\varphi^{-}(x)\right) \quad \text { for every } 1 \leq j \leq N,
$$

and

$$
\left(\Gamma_{1}, \ldots, \Gamma_{d}(t, x)\right)=\gamma(t, x)
$$

So, by (4.14),

$$
\begin{aligned}
\lim _{\varepsilon \rightarrow 0} & \int_{\Omega} \frac{1}{\varepsilon} W\left(\Psi_{\varepsilon}(x), u(x)\right) d x \\
= & \int_{J_{\varphi}}\left\{\int_{-\infty}^{0} H\left(p(t, x) \cdot\left(\varphi^{+}(x)-\varphi^{-}(x)\right) \otimes \boldsymbol{\nu}(x), \gamma(t, x), u^{+}(x)\right) d t\right. \\
& \left.+\int_{0}^{\infty} H\left(p(t, x) \cdot\left(\varphi^{+}(x)-\varphi^{-}(x)\right) \otimes \boldsymbol{\nu}(x), \gamma(t, x), u^{-}(x)\right) d t\right\} d \mathcal{H}^{N-1}(x) .
\end{aligned}
$$

Next, using the assumptions about $H$ and $\varphi$, by (4.11), we get

$$
\begin{aligned}
& \int_{\Omega} \frac{1}{\varepsilon} \mid W\left(\Psi_{\varepsilon}(x)\right., u(x))-H\left(\varepsilon \nabla \psi_{\varepsilon}(x), \psi_{\varepsilon}(x), u(x)\right) \mid d x \\
& \leq C \int_{B_{R}(0)} \int_{\Omega}|\bar{\varphi}(x+\varepsilon z)-\bar{\varphi}(x)| d z d x \leq \bar{C} \varepsilon\|D \bar{\varphi}\|\left(\mathbb{R}^{N}\right) .
\end{aligned}
$$

Therefore, returning to (4.19), we conclude the desired result (4.4).

With the same method used in the proof of Theorem 4.1, we can prove the following generalization.

\section{Theorem 4.2. Let}

$$
\begin{aligned}
& H\left(a_{1}, a_{2}, \ldots, a_{k-1}, a_{k}, b, c\right): \\
& \quad\left\{\mathbb{R}^{d \times N^{k}} \times \mathbb{R}^{d \times N^{k-1}} \times \cdots \times \mathbb{R}^{d \times N \times N} \times \mathbb{R}^{d \times N}\right\} \times \mathbb{R}^{d} \times \mathbb{R}^{q} \rightarrow \mathbb{R}
\end{aligned}
$$

be a $C^{1}$-function satisfying $H \geq 0$. Consider $u \in B V\left(\Omega, \mathbb{R}^{q}\right) \cap L^{\infty}$ and $\varphi \in$ $B V\left(\Omega, \mathbb{R}^{d}\right) \cap L^{\infty}$ satisfying $H(0, \ldots, 0, \varphi(x), u(x))=0$ for a.e. $x \in \Omega$. For any $\eta \in \mathcal{V} \cap C^{k+1}\left(\mathbb{R}^{N} \times \mathbb{R}^{N}, \mathbb{R}^{d \times d}\right)$, let $\psi_{\varepsilon}$ be defined by (4.2). Then

$$
\begin{aligned}
\lim _{\varepsilon \rightarrow 0} & \int_{\Omega} \frac{1}{\varepsilon} H\left(\varepsilon^{k} \nabla^{k} \psi_{\varepsilon}, \varepsilon^{k-1} \nabla^{k-1} \psi_{\varepsilon}, \ldots, \varepsilon^{2} \nabla^{2} \psi_{\varepsilon}, \varepsilon \nabla \psi_{\varepsilon}, \psi_{\varepsilon}, u\right) d x \\
= & \int_{J_{\varphi}}\left\{\int_{-\infty}^{0} H\left(\iota_{k}(t, x), \iota_{k-1}(t, x), \ldots, \iota_{2}(t, x), \iota_{1}(t, x), \gamma(t, x), u^{+}(x)\right) d t\right. \\
& \left.\quad+\int_{0}^{\infty} H\left(\iota_{k}(t, x), \iota_{k-1}(t, x), \ldots, \iota_{2}(t, x), \iota_{1}(t, x), \gamma(t, x), u^{-}(x)\right) d t\right\} d \mathcal{H}^{N-1}(x),
\end{aligned}
$$


where $p$ and $\gamma$ are defined in (3.6) and (3.5), respectively,

$$
\iota_{i}(t, x):=(-1)^{i-1} \frac{\partial^{i-1}}{\partial t^{i-1}} p(t, x) \cdot\left(\varphi^{+}(x)-\varphi^{-}(x)\right) \bigotimes_{j=1}^{i} \boldsymbol{\nu}(x),
$$

and it is assumed that the orientation of $J_{u}$ coincides with the orientation of $J_{\varphi}$ $\mathcal{H}^{N-1}$ a.e. on $J_{u} \cap J_{\varphi}$.

\section{Optimizing the upper bound}

We want to optimize the bounds obtained in Theorems 4.1 and 4.2 on the choice of the kernels. Let $H, \varphi: \Omega \rightarrow \mathbb{R}^{d}$ and $u: \Omega \rightarrow \mathbb{R}^{q}$ be as in Theorem 4.1. Define $Z: \mathcal{V} \rightarrow \mathbb{R}$ by

$$
\begin{aligned}
& Z(\eta)=\int_{J_{\varphi}}\left\{\int_{-\infty}^{0} H\left(p(t, x) \cdot\left(\varphi^{+}(x)-\varphi^{-}(x)\right) \otimes \boldsymbol{\nu}(x), \gamma(t, x), u^{+}(x)\right) d t\right. \\
& \left.+\int_{0}^{\infty} H\left(p(t, x) \cdot\left(\varphi^{+}(x)-\varphi^{-}(x)\right) \otimes \boldsymbol{\nu}(x), \gamma(t, x), u^{-}(x)\right) d t\right\} d \mathcal{H}^{N-1}(x)
\end{aligned}
$$

where $\boldsymbol{\nu}(x)$ is the jump vector of $\varphi$ and $p$ and $\gamma$ are defined in (3.6) and (3.5), respectively. We require the following result.

Theorem 5.1. Let $H, u$ and $\varphi$ be as in Theorem 4.1. Let $Z$ be as in (5.1). Then

$$
\begin{aligned}
\inf _{\eta \in \mathcal{V}} Z(\eta)=\mathcal{J}_{0}(\varphi):= & \int_{J_{\varphi}}\left(\operatorname { i n f } _ { r \in \mathcal { R } _ { \varphi ^ { + } ( x ) , \varphi ^ { - } ( x ) } ^ { ( 0 ) } } \left\{\int_{-\infty}^{0} H\left(-r^{\prime}(t) \otimes \boldsymbol{\nu}(x), r(t), u^{+}(x)\right) d t\right.\right. \\
& \left.\left.+\int_{0}^{\infty} H\left(-r^{\prime}(t) \otimes \boldsymbol{\nu}(x), r(t), u^{-}(x)\right) d t\right\}\right) d \mathcal{H}^{N-1}(x),
\end{aligned}
$$

where $\mathcal{R}_{\boldsymbol{a}, \boldsymbol{b}}^{(0)}$ is defined by

$$
\mathcal{R}_{\boldsymbol{a}, \boldsymbol{b}}^{(0)}:=\left\{r(t) \in C^{1}\left(\mathbb{R}, \mathbb{R}^{d}\right): \exists L>0 \text { s.t. } r(t)=\boldsymbol{a} \text { for } t \leq-L, r(t)=\boldsymbol{b} \text { for } t \geq L\right\} .
$$

Moreover, if $H(a, b, c)=|a|^{2}+P(b, c)$ for some $P \in C^{1}$, then

$$
\begin{aligned}
\inf _{\eta \in \mathcal{V}} Z(\eta)=\int_{J_{\varphi}}\left(\inf _{r \in \mathcal{R}_{\varphi^{+}(x), \varphi^{-}(x)}}\right. & \left\{\int_{-1}^{0}\left|r^{\prime}(t)\right| \sqrt{P\left(r(t), u^{+}(x)\right)} d t\right. \\
+ & \left.\left.\int_{0}^{1}\left|r^{\prime}(t)\right| \sqrt{P\left(r(t), u^{-}(x)\right)} d t\right\}\right) d \mathcal{H}^{N-1}(x),
\end{aligned}
$$

where $\mathcal{R}_{\boldsymbol{a}, \boldsymbol{b}}:=\left\{r(t) \in C^{1}\left([-1,1], \mathbb{R}^{d}\right): r(-1)=\boldsymbol{a}, r(1)=\boldsymbol{b}\right\}$. 
Before proving Theorem 5.1, we show how it implies the main theorem of this work on first order problems.

Proof. For $\eta \in \mathcal{V}$, consider $Z(\eta)$ as in (5.1). By Theorem 5.1, there exists a sequence $\left\{\eta_{n}\right\} \subset \mathcal{V}$ such that

$$
0 \leq Z\left(\eta_{n}\right)-\mathcal{J}_{0}(\varphi) \leq 1 / n, \quad \text { for all } n \geq 1 .
$$

By Corollary 4.1, for any $\eta_{n} \in \mathcal{V}$ and $0<\varepsilon<1$, there exists $\psi_{\varepsilon, n} \in C^{2}\left(\mathbb{R}^{N}, \mathbb{R}^{d}\right)$ such that $\int_{\Omega} \psi_{\varepsilon, n}(x) d x=\int_{\Omega} \varphi(x) d x$,

$$
\lim _{\varepsilon \rightarrow 0^{+}} \psi_{\varepsilon, n}=\varphi \quad \text { in } \quad L^{p}\left(\Omega, \mathbb{R}^{d}\right) \quad \text { for all } p \in[1, \infty),
$$

and

$$
\lim _{\varepsilon \rightarrow 0} \int_{\Omega} \frac{1}{\varepsilon} H\left(\varepsilon \nabla \psi_{\varepsilon, n}, \psi_{\varepsilon, n}, u\right) d x=Z\left(\eta_{n}\right) .
$$

A standard diagonal argument concludes the proof. We include it for the reader's convenience. Define a positive sequence $\left\{\varepsilon_{n}\right\}_{n=0}^{\infty}$ as follows. Set $\varepsilon_{0}=1$. Assuming that $\varepsilon_{n-1}$ has already been defined, choose $0<\varepsilon_{n}<\min \left\{\varepsilon_{n-1}, 1 / n\right\}$ such that for every $\varepsilon<\varepsilon_{n}$, we have

$$
\left(\int_{\Omega}\left|\psi_{\varepsilon, n}(x)-\varphi(x)\right|^{n} d x\right)^{1 / n}<\frac{1}{n}
$$

and

$$
\left|\int_{\Omega} \frac{1}{\varepsilon} H\left(\varepsilon \nabla \psi_{\varepsilon, n}, \psi_{\varepsilon, n}, u\right) d x-Z\left(\eta_{n}\right)\right|<\frac{1}{n} .
$$

Thus, $\bigcup_{n=0}^{\infty}\left[\varepsilon_{n+1}, \varepsilon_{n}\right)=(0,1)$. For any $\varepsilon \in(0,1)$ let $k$ be the unique integer such that $\varepsilon \in\left[\varepsilon_{k+1}, \varepsilon_{k}\right)$, and then define $\psi_{\varepsilon}(x)=\psi_{\varepsilon, k}(x)$. By (5.2), (5.3) and (5.4), for every $n \geq 1$, we have

$$
\left(\int_{\Omega}\left|\psi_{\varepsilon}(x)-\varphi(x)\right|^{n} d x\right)^{1 / n}<\frac{1}{n} \quad \text { for all } \varepsilon<\varepsilon_{n}
$$

and

$$
\left|\lim _{\varepsilon \rightarrow 0} \int_{\Omega} \frac{1}{\varepsilon} H\left(\varepsilon \nabla \psi_{\varepsilon}, \psi_{\varepsilon}, u\right) d x-\mathcal{J}_{0}(\varphi)\right|<\frac{2}{n} \quad \text { for all } \varepsilon<\varepsilon_{n} .
$$

In particular, by Hölder's inequality, we obtain for every $p \leq n / 2$,

$$
\begin{aligned}
& \left(\int_{\Omega}\left|\psi_{\varepsilon}(x)-\varphi(x)\right|^{p} d x\right)^{1 / p} \leq \\
& \quad\left(\int_{\Omega}\left|\psi_{\varepsilon}(x)-\varphi(x)\right|^{n} d x\right)^{1 / n}|\Omega|^{\frac{1}{p(1-p / n)}}<\frac{|\Omega|^{2 / p}+1}{n} \text { for all } \varepsilon<\varepsilon_{n} .
\end{aligned}
$$

This completes the proof. 
We now come to the proof of Theorem 5.1. First we need the following technical statements.

Lemma 5.1. Let $\mu$ be a finite positive Borel measure on $\Omega$ and $\nu_{0}: \Omega \rightarrow \mathbb{R}^{N}$ a Borel measurable function with $\left|\nu_{0}\right|=1$. Let $\mathcal{W}$ denote the set of functions $p(t, x): \mathbb{R} \times \Omega \rightarrow \mathbb{R}^{d \times d}$ satisfying the following conditions:

i) $p$ is Borel measurable and bounded;

ii) there exists $M>0$ such that $p(t, x)=0$ for $|t|>M$ and any $x \in \Omega$;

iii) $\int_{\mathbb{R}} p(t, x) d t=I$ for all $x \in \Omega$.

Then, for every $p \in \mathcal{W}$, there exists a sequence of functions $\left\{\eta_{n}\right\} \subset \mathcal{V}$ (see Definition 4.1) such that the sequence of functions $\left\{p_{n}(t, x)\right\}$ defined on $\mathbb{R} \times \Omega$ by

$$
p_{n}(t, x)=\int_{H_{\nu_{0}(x)}^{0}} \eta_{n}\left(t \boldsymbol{\nu}_{0}(x)+y, x\right) d \mathcal{H}^{N-1}(y),
$$

has the following properties:

i) there exists $C_{0}$ such that $\left\|p_{n}\right\|_{L^{\infty}} \leq C_{0}$ for every $n$;

ii) there exists $M>0$ such that for all $n$, we have $p_{n}(t, x)=0$ for $|t|>M$ and every $x \in \Omega$;

iii) $\lim _{n \rightarrow \infty} \int_{\Omega} \int_{\mathbb{R}}\left|p_{n}(t, x)-p(t, x)\right| d t d \mu(x)=0$.

See the Appendix for the proof of this lemma.

Lemma 5.2. Let $\mu$ be a finite positive Borel measure on $\Omega, \nu_{0}: \Omega \rightarrow \mathbb{R}^{N}$ a Borel measurable function, with $\left|\nu_{0}\right|=1$ and $h: \Omega \rightarrow G \subset \mathbb{R}^{l}$ a Borel measurable function. Let $L_{1}(A, B, s) \in C\left(\mathbb{R}^{d \times d} \times \mathbb{R}^{d \times d} \times G, \mathbb{R}\right)$ and $L_{2}(A, B, s) \in$ $C\left(\mathbb{R}^{d \times d} \times \mathbb{R}^{d \times d} \times G, \mathbb{R}\right)$ satisfy $L_{j} \geq 0$ for $j=1,2, L_{1}(O, O, h(x))=0$ for $\mu$-a.e. $x$ in $\Omega$ and $L_{2}(O, I, h(x))=0$ for $\mu$-a.e. $x$ in $\Omega$. Suppose also that for every bounded set $\mathcal{A} \subset \mathbb{R}^{d \times d}$ there exists $C=C_{\mathcal{A}}>0$ such that for $j=1,2$ we have

$$
\begin{aligned}
& \left|L_{j}\left(A_{1}, B_{1}, h(x)\right)-L_{j}\left(A_{2}, B_{2}, h(x)\right)\right| \leq C_{\mathcal{A}}\left(\left|A_{1}-A_{2}\right|+\left|B_{1}-B_{2}\right|\right) \\
& \text { for } \mu \text {-a.e. } x \in \Omega \text { and every } A_{1}, A_{2}, B_{1}, B_{2} \in \mathcal{A} .
\end{aligned}
$$

Define $Y: \mathcal{V} \rightarrow \mathbb{R}$ by

$$
\begin{aligned}
Y(\eta)=\int_{\Omega}\left\{\int_{-\infty}^{0} L_{1}\left(p(t, x), \int_{-\infty}^{t} p(s, x) d s, h(x)\right) d t\right. \\
\left.\quad+\int_{0}^{+\infty} L_{2}\left(p(t, x), \int_{-\infty}^{t} p(s, x) d s, h(x)\right) d t\right\} d \mu(x)
\end{aligned}
$$

where $p$ is defined by

$$
p(t, x)=\int_{H_{\nu_{0}(x)}^{0}} \eta\left(t \boldsymbol{\nu}_{0}(x)+y, x\right) d \mathcal{H}^{N-1}(y) .
$$


Then

$$
\inf _{\eta \in \mathcal{V}} Y(\eta)=\int_{\Omega}\left\{\inf _{p \in \mathcal{P}} Q_{x}(p)\right\} d \mu(x)
$$

where

$$
\mathcal{P}=\left\{p \in L_{c}^{\infty}\left(\mathbb{R}, \mathbb{R}^{d \times d}\right): \int_{\mathbb{R}} p(t) d t=I\right\}
$$

and

$$
Q_{x}(p)=\int_{-\infty}^{0} L_{1}\left(p(t), \int_{-\infty}^{t} p(s) d s, h(x)\right) d t+\int_{0}^{+\infty} L_{2}\left(p(t), \int_{-\infty}^{t} p(s) d s, h(x)\right) d t .
$$

Proof. Clearly,

$$
\inf _{\eta \in \mathcal{V}} Y(\eta) \geq \int_{J_{\varphi}}\left\{\inf _{p \in \mathcal{P}} Q_{x}(p)\right\} d \mu(x)
$$

so (5.6) follows once we prove the reverse inequality. In the proof, we establish two claims.

Claim 1: The function $\zeta(x):=\inf _{p \in \mathcal{P}} Q_{x}(p)$, for all $x \in \Omega$, is Borel measurable.

Consider the countable subset $\mathcal{P}_{r} \subset \mathcal{P}$ defined by

$$
\begin{array}{r}
\mathcal{P}_{r}:=\left\{p \in \mathcal{P}: p(t)=\alpha_{0}+\alpha_{1} t+\cdots+\alpha_{m} t^{m} \text { for }|t| \leq l \text { and } p(t)=0 \text { for }|t|>l,\right. \\
\text { for some } \left.m, l \in \mathbb{N} \text { and } \alpha_{0}, \alpha_{1}, \ldots, \alpha_{m} \in \mathbb{Q}^{d \times d}\right\} .
\end{array}
$$

Clearly, for any $p \in \mathcal{P}$, there exists a sequence $\left\{p_{n}\right\} \subset \mathcal{P}_{r}$ and a number $M>0$ such that $p_{n} \rightarrow p$ in $L^{2}(\mathbb{R}, \mathbb{R}), \operatorname{supp} p \subset[-M, M]$ and $\operatorname{supp} p_{n} \subset[-M, M]$ for all $n$. Therefore, if $Q_{x}(p)<t$, for some $t \in \mathbb{R}$ and $x \in \Omega$, then there exists $p_{r} \in \mathcal{P}_{r}$ such that $Q_{x}\left(p_{r}\right)<t$. Thus,

$$
\{x \in \Omega: \zeta(x)<t\}=\bigcup_{p \in \mathcal{P}}\left\{x \in \Omega: Q_{x}(p)<t\right\}=\bigcup_{p \in \mathcal{P}_{r}}\left\{x \in \Omega: Q_{x}(p)<t\right\},
$$

and the measurability of $\zeta(x)$ follows. This completes the proof of Claim 1 .

Let $\mathcal{W}$ denote set of functions $p: \mathbb{R} \times \Omega \rightarrow \mathbb{R}^{d \times d}$ satisfying the following conditions:

i) $p$ is Borel measurable;

ii) $p$ is bounded on $\mathbb{R} \times \Omega$;

iii) there exists $M>0$ such that $p(t, x)=0$ for $|t|>M$ and $x \in \Omega$;

iv) $\int_{\mathbb{R}} p(t, x) d t=I$, for all $x \in \Omega$. 


\section{Claim 2:}

$$
\int_{\Omega}\left\{\inf _{p \in \mathcal{P}} Q_{x}(p)\right\} d \mu(x)=\inf _{p \in \mathcal{W}}\left\{\int_{\Omega} Q_{x}(p(t, x)) d \mu(x)\right\} .
$$

Fix a bounded Borel measurable function $p \in \mathcal{P}$. We have

$$
Q_{x}(p) \leq D, \quad \text { for } \mu \text {-a.e. } x \in \Omega,
$$

where $D>0$ does not depend on $x$. Next fix $\varepsilon>0$. By Lusin's Theorem, there exists a compact set $K \subset \Omega$ such that $h(x)$ and $\zeta(x)$ are continuous functions on $K$ and

$$
\mu(\Omega \backslash K) \leq \frac{\varepsilon}{2 D}
$$

Here $\zeta$ is the function given by Claim 1. For any $x \in K$, there exists $p_{x} \in \mathcal{P}$ such that $p_{x}(t)$ is a bounded Borel measurable function on $\mathbb{R}$ and

$$
Q_{x}\left(p_{x}\right)-\zeta(x)<\frac{\varepsilon}{4+4 \mu(\Omega)} .
$$

Using the continuity of $\zeta$ and $h$ on $K$, we infer from (5.11) that for any $x \in K$, there exists $\delta_{x}>0$ such that

$$
Q_{y}\left(p_{x}\right)-\zeta(y)<\frac{\varepsilon}{2+2 \mu(\Omega)}, \quad \text { for all } y \in K \cap B_{\delta_{x}}(x) .
$$

Since the set $K$ is compact, there exist a finite number of points $x_{1}, x_{2}, \ldots, x_{l} \in K$ such that $K \subset \bigcup_{j=1}^{l} B_{\delta_{x_{j}}}\left(x_{j}\right)$. Define the function $\bar{p}(t, x)$ on $\mathbb{R} \times \Omega$ by

$$
\bar{p}(t, x)= \begin{cases}p_{x_{i}}(t) & x \in\left(K \bigcap B_{\delta_{x_{i}}}(x)\right) \backslash \bigcup_{1 \leq j \leq i-1} B_{\delta_{x_{j}}}\left(x_{j}\right) \quad 1 \leq i \leq l, \\ p(t) & x \in \Omega \backslash K .\end{cases}
$$

Clearly, $\bar{p}(t, x) \in \mathcal{W}$. From (5.12) and (5.9)-(5.10), we get

$$
\int_{\Omega} Q_{x}(\bar{p}(t, x)) \mu(x)-\int_{\Omega} \zeta(x) d \mu(x)<\varepsilon,
$$

which implies (5.8), since $\varepsilon>0$ is arbitrary.

We are now ready to complete the proof of Lemma 5.2. Consider a function $p(t, x) \in \mathcal{W}$, and let $\eta_{n}(t, x) \in \mathcal{V}$ and $p_{n}(t, x)$ be the corresponding functions given by Lemma 5.1 . We have

$$
\begin{aligned}
& Y\left(\eta_{n}\right)-\int_{\Omega} Q_{x}(p(t, x)) d \mu(x) \\
& \leq C \int_{\Omega}\left(\int_{-c}^{c}\left\{\left|p_{n}(t, \cdot)-p(t, \cdot)\right|+\int_{-c}^{c}\left|p_{n}(s, \cdot)-p(s, \cdot)\right| d s\right\} d t\right) d \mu
\end{aligned}
$$


for some constants $c, C>0$. Therefore, using (A.8) and (5.13), we conclude that

$$
\varlimsup_{n \rightarrow \infty} Y\left(\eta_{n}\right) \leq \int_{\Omega} Q_{x}(p(t, x)) d \mu(x) .
$$

Since this holds for every $p(t, x) \in \mathcal{W}$, we get

$$
\inf _{\eta \in \mathcal{V}} Y(\eta)=\inf _{p \in \mathcal{W}}\left\{\int_{\Omega} Q_{x}(p(t, x)) d \mu(x)\right\} .
$$

Combining (5.14) with Claim 2, we obtain the desired result (5.6).

Lemma 5.3. Let $\Theta(x, y), \Xi(x, y) \in C^{1}\left(\mathbb{R}^{d} \times \mathbb{R}^{d}, \mathbb{R}\right)$ satisfy $\Theta \geq 0, \Xi \geq 0$ and $\Theta(0, \boldsymbol{a})=\Xi(0, \boldsymbol{b})=0$, for some $\boldsymbol{a} \neq \boldsymbol{b}$ in $\mathbb{R}^{d}$. Set

$$
\mathcal{P}=\left\{p \in L_{c}^{\infty}\left(\mathbb{R}, \mathbb{R}^{d \times d}\right): \int_{\mathbb{R}} p(t) d t=I\right\}
$$

and define $U: \mathcal{P} \rightarrow \mathbb{R}$ by

$$
\begin{aligned}
U(p)= & \int_{-\infty}^{0} \Theta\left(p(t) \cdot(\boldsymbol{b}-\boldsymbol{a}),\left(\int_{-\infty}^{t} p(s) d s\right) \cdot \boldsymbol{b}+\left(\int_{t}^{+\infty} p(s) d s\right) \cdot \boldsymbol{a}\right) d t \\
& +\int_{0}^{+\infty} \Xi\left(p(t) \cdot(\boldsymbol{b}-\boldsymbol{a}),\left(\int_{-\infty}^{t} p(s) d s\right) \cdot \boldsymbol{b}+\left(\int_{t}^{+\infty} p(s) d s\right) \cdot \boldsymbol{a}\right) d t
\end{aligned}
$$

Then

$$
\inf _{p \in \mathcal{P}} U(p)=\inf _{r \in \mathcal{R}_{\boldsymbol{a}, \boldsymbol{b}}^{(0)}} I_{0}(r)
$$

where

$$
\mathcal{R}_{\boldsymbol{a}, \boldsymbol{b}}^{(0)}:=\left\{r(t) \in C^{1}\left(\mathbb{R}, \mathbb{R}^{d}\right): \exists L>0 \text { s.t. } r(t)=\boldsymbol{a} \text { for } t \leq-L, r(t)=\boldsymbol{b} \text { for } t \geq L\right\}
$$

and $I_{0}(r): \mathcal{R}_{\boldsymbol{a}, \boldsymbol{b}}^{(0)} \rightarrow \mathbb{R}$ is defined by

$$
I_{0}(r):=\int_{-\infty}^{0} \Theta\left(r^{\prime}(t), r(t)\right) d t+\int_{0}^{+\infty} \Xi\left(r^{\prime}(t), r(t)\right) d t .
$$

If, in addition, $\Theta(x, y)=|x|^{2}+F(y)$ and $\Xi(x, y)=|x|^{2}+G(y)$ for some $F, G \in C^{1}$, then

$$
\inf _{p \in \mathcal{P}} U(p)=\inf _{r \in \mathcal{R}_{\boldsymbol{a}, \boldsymbol{b}}} I(r),
$$

where $\mathcal{R}_{\boldsymbol{a}, \boldsymbol{b}}:=\left\{r(t) \in C^{1}\left([-1,1], \mathbb{R}^{d}\right): r(-1)=\boldsymbol{a}, r(1)=\boldsymbol{b}\right\}$ and

$$
I(r):=\int_{-1}^{0} 2\left|r^{\prime}(t)\right| \sqrt{F(r(t))} d t+\int_{0}^{1} 2\left|r^{\prime}(t)\right| \sqrt{G(r(t))} d t .
$$


Proof. It is clear by density arguments that

$$
\inf _{p \in \mathcal{P}} U(p)=\inf _{p \in \mathcal{P} \cap C_{c}\left(\mathbb{R}, \mathbb{R}^{d \times d}\right)} U(p) .
$$

For any $p(t) \in \mathcal{P} \cap C_{c}\left(\mathbb{R}, \mathbb{R}^{d \times d}\right)$, consider

$$
r_{p}(t):=\left(\int_{-\infty}^{t} p(s) d s\right) \cdot \boldsymbol{b}+\left(\int_{t}^{+\infty} p(s) d s\right) \cdot \boldsymbol{a} .
$$

Then $r_{p} \in \mathcal{R}_{\boldsymbol{a}, \boldsymbol{b}}^{(0)}$ and $U(p)=I_{0}\left(r_{p}\right)$. Therefore, by (5.20), we infer that

$$
\inf _{p \in \mathcal{P}} U(p) \geq \inf _{r \in \mathcal{R}_{a, b}^{(0)}} I_{0}(r)
$$

On the other hand, let $\left(r_{1}(t), \ldots, r_{k}(t)\right):=r(t) \in \mathcal{R}_{\boldsymbol{a}, \boldsymbol{b}}^{(0)}$ and let $L>0$ be as in (5.16). Define the function $p_{r}(t): \mathbb{R} \rightarrow \mathbb{R}^{d \times d}$ in the following way. Since $\left(b_{1}, \ldots, b_{d}\right):=$ $\boldsymbol{b} \neq\left(a_{1}, \ldots, a_{d}\right):=\boldsymbol{a}$, there exists $k \in\{1,2, \ldots, d\}$, which we fix throughout this argument, such that $b_{k} \neq a_{k}$. Fix also some function $\varpi(t) \in L^{\infty}([-L, L], \mathbb{R})$ satisfying $\int_{-L}^{L} \varpi(t) d t=1$. For $|t| \leq L$ let $p_{i j}^{(r)}$ be the $i j$-th entry of matrix $p_{r}(t)$. Then define

$$
\begin{aligned}
& p_{i i}^{(r)}(t):=\left\{\begin{array}{l}
\frac{1}{b_{i}-a_{i}} r_{i}^{\prime}(t) \text { if } b_{i} \neq a_{i}, \\
\varpi(t) \quad \text { if } b_{i}=a_{i},
\end{array} \quad \text { for } i \in\{1,2, \ldots, d\},\right. \\
& p_{i k}^{(r)}:=\left\{\begin{array}{l}
0 \quad \text { if } b_{i} \neq a_{i}, \\
\frac{1}{b_{k}-a_{k}} r_{i}^{\prime}(t) \text { if } b_{i}=a_{i},
\end{array} \quad \text { for } i \neq k,\right. \\
& p_{i j}^{(r)}(t)=0 \quad \text { if } j \neq i, k \text {. }
\end{aligned}
$$

Define also $p_{r}(t)=O$ for any $|t|>L$. Then $p_{r}(t) \in \mathcal{P}$. Moreover, $p(t) \cdot(\boldsymbol{b}-\boldsymbol{a})=$ $r^{\prime}(t)$; and therefore, $\left(\int_{-\infty}^{t} p(s) d s\right) \cdot \boldsymbol{b}+\left(\int_{t}^{+\infty} p(s) d s\right) \cdot \boldsymbol{a}=r(t)$. So $U\left(p_{r}\right)=I_{0}(r)$. Hence

$$
\inf _{p \in \mathcal{P}} U(p) \leq \inf _{r \in \mathcal{R}_{\boldsymbol{a}, \boldsymbol{b}}^{(0)}} I_{0}(r)
$$

Thus, from (5.21), we infer (5.15).

Now let $\Theta(x, y)=|x|^{2}+F(y)$ and $\Xi(x, y)=|x|^{2}+G(y)$ for some $F, G \in C^{1}$. For any $r \in \mathcal{R}_{\boldsymbol{a}, \boldsymbol{b}}^{(0)}$, define $\bar{r}:[-1,1] \rightarrow \mathbb{R}$ by

$$
\bar{r}(t):=r(L t)
$$

where $L>0$ is as in (5.16). Then $\bar{r} \in \mathcal{R}_{\boldsymbol{a}, \boldsymbol{b}}$ and, by the inequality $a^{2}+b^{2} \geq 2 a b$, 
we have

$$
\begin{aligned}
I_{0}(r) & =\int_{-L}^{0}\left\{\left|r^{\prime}(t)\right|^{2}+F(r(t))\right\} d t+\int_{0}^{L}\left\{\left|r^{\prime}(t)\right|^{2}+G(r(t))\right\} d t \\
& \geq \int_{-L}^{0} 2\left|r^{\prime}(t)\right| \sqrt{F(r(t))} d t+\int_{0}^{L} 2\left|r^{\prime}(t)\right| \sqrt{G(r(t))} d t=I(\bar{r}) .
\end{aligned}
$$

Therefore, from (5.15), we infer

$$
\inf _{p \in \mathcal{P}} U(p) \geq \inf _{r \in \mathcal{R}_{\boldsymbol{a}, \boldsymbol{b}}} I(r)
$$

Now let $r(t) \in \mathcal{R}_{\boldsymbol{a}, \boldsymbol{b}}$, such that $r^{\prime}(t) \neq 0$ for any $t \in[-1,1]$. Define $\tau_{n}(t) \in$ $C(\mathbb{R},[-1,1])$ as the solution of

$$
\left\{\begin{array}{l}
\tau_{n}^{\prime}(t)=\frac{\sqrt{F\left(r\left(\tau_{n}(t)\right)\right)}}{\left|r^{\prime}\left(\tau_{n}(t)\right)\right|}+\frac{1}{n}\left(1+\tau_{n}(t)\right) \quad t \in(-\infty, 0), \\
\tau_{n}^{\prime}(t)=\frac{\sqrt{G\left(r\left(\tau_{n}(t)\right)\right)}}{\left|r^{\prime}\left(\tau_{n}(t)\right)\right|}+\frac{1}{n}\left(1-\tau_{n}(t)\right) \quad t \in(0,+\infty), \\
\tau_{n}(0)=0 .
\end{array}\right.
$$

Then $\tau_{n} \in \operatorname{Lip}(\mathbb{R}), \tau_{n}$ is non-decreasing on $\mathbb{R}$ and $\lim _{t \rightarrow-\infty} \tau_{n}(t)=-1, \lim _{t \rightarrow+\infty} \tau_{n}(t)=1$. Moreover,

$$
0 \leq 1-\left|\tau_{n}(t)\right| \leq e^{-|t| / n}, \quad t \in \mathbb{R}
$$

Next for any $n \in \mathbb{N}$, define $\tilde{r}_{n}(t): \mathbb{R} \rightarrow \mathbb{R}^{d}$ by $\tilde{r}_{n}(t):=r\left(\tau_{n}(t)\right)$. Then

$$
I_{0}\left(\tilde{r}_{n}\right)=\int_{-\infty}^{+\infty}\left(\tau_{n}^{\prime}(t)\right)^{2}\left|r^{\prime}\left(\tau_{n}(t)\right)\right|^{2}+\int_{-\infty}^{0} F\left(r\left(\tau_{n}(t)\right)\right) d t+\int_{0}^{+\infty} G\left(r\left(\tau_{n}(t)\right)\right) d t .
$$


Therefore, by (5.23),

$$
\begin{aligned}
I_{0}\left(\tilde{r}_{n}\right)= & \int_{-\infty}^{0} \tau_{n}^{\prime}(t)\left|r^{\prime}\left(\tau_{n}(t)\right)\right|\left(\sqrt{F\left(r\left(\tau_{n}(t)\right)\right)}+\frac{1}{n}\left(1+\tau_{n}(t)\right)\left|r^{\prime}\left(\tau_{n}(t)\right)\right|\right) d t \\
& +\int_{0}^{+\infty} \tau_{n}^{\prime}(t)\left|r^{\prime}\left(\tau_{n}(t)\right)\right|\left(\sqrt{G\left(r\left(\tau_{n}(t)\right)\right)}+\frac{1}{n}\left(1-\tau_{n}(t)\right)\left|r^{\prime}\left(\tau_{n}(t)\right)\right|\right) d t \\
& +\int_{-\infty}^{0}\left(\tau_{n}^{\prime}(t)\left|r^{\prime}\left(\tau_{n}(t)\right)\right|-\frac{1}{n}\left(1+\tau_{n}(t)\right)\left|r^{\prime}\left(\tau_{n}(t)\right)\right|\right) \sqrt{F\left(r\left(\tau_{n}(t)\right)\right)} d t \\
& +\int_{0}^{+\infty}\left(\tau_{n}^{\prime}(t)\left|r^{\prime}\left(\tau_{n}(t)\right)\right|-\frac{1}{n}\left(1-\tau_{n}(t)\right)\left|r^{\prime}\left(\tau_{n}(t)\right)\right|\right) \sqrt{G\left(r\left(\tau_{n}(t)\right)\right)} d t \\
= & I(r)+\frac{1}{n} \int_{-\infty}^{0}\left(1+\tau_{n}(t)\right)\left|r^{\prime}\left(\tau_{n}(t)\right)\right|\left(\tau_{n}^{\prime}(t)\left|r^{\prime}\left(\tau_{n}(t)\right)\right|-\sqrt{F\left(r\left(\tau_{n}(t)\right)\right)}\right) d t \\
& +\frac{1}{n} \int_{0}^{+\infty}\left(1-\tau_{n}(t)\right)\left|r^{\prime}\left(\tau_{n}(t)\right)\right|\left(\tau_{n}^{\prime}(t)\left|r^{\prime}\left(\tau_{n}(t)\right)\right|-\sqrt{\left.G\left(r\left(\tau_{n}(t)\right)\right)\right)} d t\right. \\
= & I(r)+\frac{1}{n^{2}} \int_{-\infty}^{+\infty}\left(1-\left|\tau_{n}(t)\right|\right)^{2}\left|r^{\prime}\left(\tau_{n}(t)\right)\right|^{2} d t=I(r)+O\left(\frac{1}{n}\right),
\end{aligned}
$$

where we infer the last equality from (5.24). Next, for $m>0$, define the function $\tilde{r}^{\{n, m\}}(t): \mathbb{R} \rightarrow \mathbb{R}^{d}$ by

$$
\tilde{r}^{\{n, m\}}(t)=\left\{\begin{array}{cc}
\tilde{r}_{n}(t)+\frac{1}{2}\left(\left(b-\tilde{r}_{n}(m)\right)-\left(a-\tilde{r}_{n}(-m)\right)\right) \frac{t}{m} & \\
+\frac{1}{2}\left(\left(b-\tilde{r}_{n}(m)\right)+\left(a-\tilde{r}_{n}(-m)\right)\right), & \text { for } t \in[-m, m], \\
a & \text { for } t \in(-\infty,-m), \\
b & \text { for } t \in(m,+\infty) .
\end{array}\right.
$$

Using (5.24), we see easily that for every $n$,

$$
\lim _{m \rightarrow+\infty} I_{0}\left(\tilde{r}^{\{n, m\}}\right)=I_{0}\left(\tilde{r}_{n}\right) .
$$

From (5.26), the density argument and the diagonal argument, it follows that there exists a sequence $\hat{r}_{n} \in \mathcal{R}_{\boldsymbol{a}, \boldsymbol{b}}^{(0)}$ such that

$$
\lim _{n \rightarrow+\infty} I_{0}\left(\hat{r}_{n}\right)=I(r)
$$

Then

$$
\inf _{p \in \mathcal{P}} U(p) \leq \inf \left\{I(r): r \in \mathcal{R}_{\boldsymbol{a}, \boldsymbol{b}}, r^{\prime}(t) \neq 0, \quad \text { for all } t \in[-1,1]\right\} .
$$

But by density arguments,

$$
\inf \left\{I(r): r \in \mathcal{R}_{\boldsymbol{a}, \boldsymbol{b}}, r^{\prime}(t) \neq 0, \quad \text { for all } t \in[-1,1]\right\}=\inf _{r \in \mathcal{R}_{\boldsymbol{a}, \boldsymbol{b}}} I(r) .
$$


Therefore,

$$
\inf _{p \in \mathcal{P}} U(p) \leq \inf _{r \in \mathcal{R}_{a, b}} I(r) .
$$

This inequality, together with (5.22), gives

$$
\inf _{p \in \mathcal{P}} U(p)=\inf _{r \in \mathcal{R}_{\boldsymbol{a}, \boldsymbol{b}}} I(r) .
$$

We are now ready to prove Theorem 5.1.

Proof of Theorem 5.1. Since $Z(\eta)$ and $\mathcal{J}_{0}(\varphi)$ do not depend on the orientation of the vector $\boldsymbol{\nu}(x)$, we may assume that the orientation of $J_{\varphi}$ is such that the function $\nu: J_{\varphi} \rightarrow S^{N-1}$ is Borel measurable (see [3, Proposition 3.69]). Then we can apply Lemma 5.2 with $\mu:=\left|\varphi^{+}-\varphi^{-}\right| \mathcal{H}^{N-1}\left\llcorner J_{\varphi}, \nu_{0}(x):=\boldsymbol{\nu}(x)\right.$, $h(x):=\left(\varphi^{+}(x), \varphi^{-}(x), u^{+}(x), u^{-}(x), \nu(x)\right)$ to infer

$$
\begin{aligned}
\inf _{\eta \in \mathcal{V}} Z(\eta) & =\int_{J_{\varphi}}\left(\operatorname { i n f } _ { p ( t ) \in \mathcal { P } } \left\{\int_{-\infty}^{0} H\left(p(t) \cdot\left(\varphi^{+}(x)-\varphi^{-}(x)\right) \otimes \boldsymbol{\nu}(x), \gamma(t), u^{+}(x)\right) d t\right.\right. \\
+ & \left.\left.\int_{0}^{\infty} H\left(p(t) \cdot\left(\varphi^{+}(x)-\varphi^{-}(x)\right) \otimes \boldsymbol{\nu}(x), \gamma(t), u^{-}(x)\right) d t\right\}\right) d \mathcal{H}^{N-1}(x),
\end{aligned}
$$

where

$$
\begin{aligned}
\gamma(t) & :=\varphi^{+}(x)-\left(\int_{-\infty}^{t} p(s) d s\right) \cdot\left(\varphi^{+}(x)-\varphi^{-}(x)\right) \\
& =\left(\int_{-\infty}^{t} p(s) d s\right) \cdot \varphi^{-}(x)+\left(\int_{t}^{\infty} p(s) d s\right) \cdot \varphi^{+}(x) .
\end{aligned}
$$

Finally we use Lemma 5.3 to deduce the desired result.

In a similar way as for Theorem 1.1, using Theorem 4.2, we can prove the following result.

Theorem 5.2. Let $\Omega, H, u$ and $\varphi$ be as in Theorem 4.2. Then there exists a family of functions $\left\{\psi_{\varepsilon}\right\} \subset C^{k+1}\left(\mathbb{R}^{N}, \mathbb{R}^{d}\right), 0<\varepsilon<1$, such that $\int_{\Omega} \psi_{\varepsilon}(x) d x=$ $\int_{\Omega} \varphi(x) d x$,

$$
\lim _{\varepsilon \rightarrow 0^{+}} \psi_{\varepsilon}(x)=\varphi(x) \text { in } L^{p}\left(\Omega, \mathbb{R}^{d}\right) \quad \text { for all } p \in[1, \infty),
$$

and

$$
\begin{aligned}
& \lim _{\varepsilon \rightarrow 0} \int_{\Omega} \frac{1}{\varepsilon} H\left(\varepsilon^{k} \nabla^{k} \psi_{\varepsilon}, \varepsilon^{k-1} \nabla^{k-1} \psi_{\varepsilon}, \ldots, \varepsilon^{2} \nabla^{2} \psi_{\varepsilon}, \varepsilon \nabla \psi_{\varepsilon}, \psi_{\varepsilon}, u\right) d x \\
& =\int_{J_{\varphi}}\left(\operatorname { i n f } _ { r \in \mathcal { R } _ { \varphi + ( x ) , \varphi ^ { - } ( x ) } ^ { ( 0 , k ) } } \left\{\int_{-\infty}^{0} H\left((-1)^{k} r^{(k)}(t), \ldots, r^{\prime \prime}(t),-r^{\prime}(t), r(t), u^{+}(x)\right) d t\right.\right. \\
& \left.\left.\quad+\int_{0}^{\infty} H\left((-1)^{k} r^{(k)}(t), \ldots, r^{\prime \prime}(t),-r^{\prime}(t), r(t), u^{-}(x)\right) d t\right\}\right) d \mathcal{H}^{N-1}(x),
\end{aligned}
$$


where

$$
\mathcal{R}_{\boldsymbol{a}, \boldsymbol{b}}^{(0, k)}:=\left\{r(t) \in C^{k}\left(\mathbb{R}, \mathbb{R}^{d}\right): \exists L>0 \text { s.t. } r(t)=\boldsymbol{a} \quad t \leq-L, r(t)=\boldsymbol{b} \quad t \geq L .\right\}
$$

and we assume that the orientation of $J_{u}$ coincides with the orientation of $J_{\varphi} \mathcal{H}^{N-1}$ a.e. on $J_{u} \cap J_{\varphi}$.

\section{The upper bound for the second order problem}

Definition 6.1. For a domain $\Omega \subset \mathbb{R}^{N}$, we define

$$
B V G\left(\Omega, \mathbb{R}^{d}\right):=\left\{v \in W^{1, \infty}\left(\Omega, \mathbb{R}^{d}\right): \nabla v \in B V\left(\Omega, \mathbb{R}^{d \times N}\right)\right\} .
$$

Definition 6.2. We say that the domain $\Omega \subset \mathbb{R}^{N}$ is an extension domain of second order if for any $v \in B V G\left(\Omega, \mathbb{R}^{d}\right)$ there exists $\bar{v} \in B V G\left(\mathbb{R}^{N}, \mathbb{R}^{d}\right)$ such that $\bar{v}(x)=v(x)$ for any $x \in \Omega$ and $\|D(\nabla \bar{v})\|(\partial \Omega)=0$.

Examples of extension domains of second order are given by $B V G$-domains. A bounded domain $\Omega \subset \mathbb{R}^{N}$ is a $B V G$-domain if for each point $x \in \partial \Omega$, there exist $r>0, \delta>0$ and a mapping $\gamma \in B V G\left(\mathbb{R}^{N-1}, \mathbb{R}\right)$ such that - after rotating and relabeling the coordinate axes if necessary — we have

$$
\Omega \cap C(x, r, \delta)=\left\{y=\left(y_{1}, y^{\prime}\right) \in \mathbb{R} \times \mathbb{R}^{N-1}: y_{1}>\gamma\left(y^{\prime}\right)\right\} \cap C(x, r, \delta),
$$

where $C(x, r, \delta):=\left\{y=\left(y_{1}, y^{\prime}\right) \in \mathbb{R} \times \mathbb{R}^{N-1}:\left|y_{1}-x_{1}\right|<\delta,\left|y^{\prime}-x^{\prime}\right|<r\right\}$. In other words, near $x, \partial \Omega$ is the graph of $B V G$ function. The proof of the following proposition is given in [23].

Proposition 6.1. Any bounded BVG-domain is an extension domain of second order.

Let $\Omega$ be a bounded $B V G$-domain or, more generally, a bounded extension domain of second order. Consider $v \in B V G\left(\Omega, \mathbb{R}^{d}\right)$ and $\eta(z, x) \in \mathcal{V}$, where $\mathcal{V}$ is defined in Definition 4.1. By Definition 6.2, we may extend $v$ to $\bar{v} \in B V G\left(\mathbb{R}^{N}, \mathbb{R}^{d}\right)$ such that $\bar{v}=v$ a.e. in $\Omega$, supp $\bar{v}$ is compact and $\|D(\nabla \bar{v})\|(\partial \Omega)=0$. For $\varepsilon>0$ and any $x \in \mathbb{R}^{N}$, set

$$
v_{\varepsilon}(x):=\frac{1}{\varepsilon^{N}} \int_{\mathbb{R}^{N}} \eta\left(\frac{y-x}{\varepsilon}, x\right) \cdot \bar{v}(y) d y=\int_{\mathbb{R}^{N}} \eta(z, x) \cdot \bar{v}(x+\varepsilon z) d z .
$$

Then $v_{\varepsilon} \in C_{c}^{2}\left(\mathbb{R}^{N}, \mathbb{R}^{d}\right)$; and for every $p \geq 1$, we have $\lim _{\varepsilon \rightarrow 0^{+}} v_{\varepsilon}=v$ in $W^{1, p}\left(\Omega, \mathbb{R}^{d}\right)$.

The next theorems correspond to the theorems of the previous sections for the second order problem. Their proofs are given below. 
Theorem 6.1. Let $\Omega$ be a bounded, Lipschitz and extension domain of second order and let $F \in C^{1}\left(\mathbb{R}^{d \times N \times N} \times \mathbb{R}^{d \times N} \times \mathbb{R}^{d} \times \mathbb{R}^{q}, \mathbb{R}\right)$, satisfying $F \geq 0$. Let $f \in B V\left(\Omega, \mathbb{R}^{q}\right) \cap L^{\infty}$ and $v \in B V G\left(\Omega, \mathbb{R}^{d}\right)$ be such that $F(0, \nabla v(x), v(x), f(x))=0$ a.e. in $\Omega$. Consider $\nabla^{2} v_{\varepsilon}:=\nabla\left(\nabla v_{\varepsilon}\right)$, where $v_{\varepsilon}$ is defined in (6.1). Then

(6.2) $\lim _{\varepsilon \rightarrow 0} \frac{1}{\varepsilon} \int_{\Omega} F\left(\varepsilon \nabla^{2} v_{\varepsilon}(x), \nabla v_{\varepsilon}(x), v_{\varepsilon}(x), f(x)\right) d x$

$$
=\int_{J_{\nabla v}}\left\{\int_{-\infty}^{0} F\left(\left\{p(t, x) \cdot\left(\nabla v^{+}(x)-\nabla v^{-}(x)\right)\right\} \otimes \boldsymbol{\nu}(x), \Gamma(t, x), v(x), f^{+}(x)\right) d t\right.
$$

$\left.+\int_{0}^{\infty} F\left(\left\{p(t, x) \cdot\left(\nabla v^{+}(x)-\nabla v^{-}(x)\right)\right\} \otimes \boldsymbol{\nu}(x), \Gamma(t, x), v(x), f^{-}(x)\right) d t\right\} d \mathcal{H}^{N-1}(x)$,

with

$$
\Gamma(t, x):=\left(\int_{-\infty}^{t} p(s, x) d s\right) \cdot \nabla v^{-}(x)+\left(\int_{t}^{\infty} p(s, x) d s\right) \cdot \nabla v^{+}(x),
$$

where $p(t, x)$ is defined in (3.6) (with $\boldsymbol{\nu}(x)$ denoting the orientation vector of $J_{\nabla v}$ ) and we assume that the orientation of $J_{f}$ coincides with the orientation of $J_{\nabla v}$ $\mathcal{H}^{N-1}$ a.e. on $J_{f} \cap J_{\nabla v}$.

Next we turn to the minimization problem of the term on the r.h.s. of (6.2), over all kernels $\eta \in \mathcal{V}$. We consider

$$
\begin{aligned}
\widetilde{Y}(\eta):= & \int_{J_{\nabla v}}\left\{\int_{-\infty}^{0} F\left(\left\{p(t, \cdot) \cdot\left(\nabla v^{+}-\nabla v^{-}\right)\right\} \otimes \nu, \Gamma(t, \cdot), v, f^{+}\right) d t\right. \\
& \left.+\int_{0}^{\infty} F\left(\left\{p(t, \cdot) \cdot\left(\nabla v^{+}-\nabla v^{-}\right)\right\} \otimes \nu, \Gamma(t, \cdot), v, f^{-}\right) d t\right\} d \mathcal{H}^{N-1} .
\end{aligned}
$$

Since $\partial_{x_{i} x_{j}}^{2} v=\partial_{x_{j} x_{i}}^{2} v$ (as measures), we obtain $\nabla v^{+}-\nabla v^{-}=\chi \otimes \nu$. Therefore, using the results of Lemma 5.2 and Lemma 5.3 we can prove the following.

Theorem 6.2. Let $\Omega, F, f$ and $v$ be as in Theorem 6.1. Let $\tilde{Y}(\eta): \mathcal{V} \rightarrow \mathbb{R}$ be defined as in (6.4). Then

$$
\begin{aligned}
& \inf _{\eta \in \mathcal{V}} \tilde{Y}(\eta)= \widetilde{\mathcal{J}}_{0}(v):= \\
& \int_{J_{\nabla v}}\left(\operatorname { i n f } _ { r \in \mathcal { R } _ { \chi , 0 } ^ { ( 0 ) } } \left\{\int_{-\infty}^{0} F\left(-r^{\prime}(t) \otimes \boldsymbol{\nu} \otimes \boldsymbol{\nu}, r(t) \otimes \boldsymbol{\nu}+\nabla v^{-}, v, f^{+}\right) d t\right.\right. \\
&\left.\left.\quad+\int_{0}^{\infty} F\left(-r^{\prime}(t) \otimes \boldsymbol{\nu} \otimes \boldsymbol{\nu}, r(t) \otimes \boldsymbol{\nu}+\nabla v^{-}, v, f^{-}\right) d t\right\}\right) d \mathcal{H}^{N-1} .
\end{aligned}
$$


Here $\chi(x)$ is given by

$$
\chi(x) \otimes \boldsymbol{\nu}(x)=\nabla v^{+}(x)-\nabla v^{-}(x),
$$

and $\mathcal{R}_{a, b}^{(0)}$ is defined, as in (5.16), by

$$
\begin{aligned}
& \mathcal{R}_{\boldsymbol{a}, \boldsymbol{b}}^{(0)}:= \\
& \quad\left\{r(t) \in C^{1}\left(\mathbb{R}, \mathbb{R}^{d}\right): \exists L>0 \text { s.t. } r(t)=\boldsymbol{a} \text { for } t \leq-L, r(t)=\boldsymbol{b} \text { for } t \geq L\right\} .
\end{aligned}
$$

Moreover, if $F(a, b, c, d)=|a|^{2}+Q(b, c, d)$ for some $Q \in C^{1}$, then

$$
\begin{aligned}
& \inf _{\eta \in \mathcal{V}} \tilde{Y}(\eta)=\int_{J_{\nabla v}}\left(\inf _{r \in \mathcal{R}_{\chi, 0}}\right.\left\{\int_{-1}^{0}\left|r^{\prime}(t)\right| \sqrt{Q\left(r(t) \otimes \boldsymbol{\nu}+\nabla v^{-}, v, f^{+}\right)} d t\right. \\
&\left.\left.\quad+\int_{0}^{1}\left|r^{\prime}(t)\right| \sqrt{Q\left(r(t) \otimes \boldsymbol{\nu}+\nabla v^{-}, v, f^{-}\right)} d t\right\}\right) d \mathcal{H}^{N-1},
\end{aligned}
$$

where $\mathcal{R}_{\boldsymbol{a}, \boldsymbol{b}}:=\left\{r(t) \in C^{1}\left([-1,1], \mathbb{R}^{d}\right): r(-1)=\boldsymbol{a}, r(1)=\boldsymbol{b}\right\}$.

Theorem 1.2 follows from Theorem 6.1 and Theorem 6.2, just as Theorem 1.1 follows from Corollary 4.1 and Theorem 5.1. We remark that the hypothesis on domain $\Omega$ can be weakened. Indeed we need only that $\Omega$ be a bounded, Lipschitz and an extension domain of second order.

Proof of Theorem 6.1. Set $\varphi(x):=\nabla v(x)$ with $i j$-th entry $\varphi_{i j}(x)$ and $\bar{\varphi}(x):=\nabla \bar{v}(x)$ with $i j$-th entry $\bar{\varphi}_{i j}(x)$. Then $\varphi \in B V\left(\Omega, \mathbb{R}^{d \times N}\right) \cap L^{\infty}, \bar{\varphi} \in$ $B V\left(\mathbb{R}^{N}, \mathbb{R}^{d \times N}\right) \cap L^{\infty}, \bar{\varphi}$ has compact support, $\bar{\varphi}=\varphi$ on $\Omega$ and $\|D \bar{\varphi}\|(\partial \Omega)=0$. Define

$$
\psi_{\varepsilon}(x):=\frac{1}{\varepsilon^{N}} \int_{\mathbb{R}^{N}} \eta\left(\frac{y-x}{\varepsilon}, x\right) \cdot \bar{\varphi}(y) d y=\int_{\mathbb{R}^{N}} \eta(z, x) \cdot \bar{\varphi}(x+\varepsilon z) d z .
$$

Consider also the $i j$-th entry of the $d \times N$ matrix $\psi_{\varepsilon}(x)$ given by $\psi_{\varepsilon, i j}(x)$. We also denote by $\psi_{\varepsilon, j}(x):=\left(\psi_{\varepsilon, 1 j}(x), \ldots, \psi_{\varepsilon, d j}(x)\right)$ the $j$-th column of the matrix $\psi_{\varepsilon}(x)$ and by $\bar{\varphi}_{j}(x):=\left(\bar{\varphi}_{1 j}(x), \ldots, \bar{\varphi}_{d j}(x)\right)$ the $j$-th column of the matrix $\bar{\varphi}(x)$. First of all, we prove that

$$
\lim _{\varepsilon \rightarrow 0} \int_{\Omega} \frac{1}{\varepsilon}\left|F\left(\varepsilon \nabla^{2} v_{\varepsilon}(x), \nabla v_{\varepsilon}(x), v_{\varepsilon}(x), f(x)\right)-F\left(\varepsilon \nabla \psi_{\varepsilon}(x), \psi_{\varepsilon}(x), v_{\varepsilon}(x), f(x)\right)\right| d x=0 .
$$




\section{We compute}

(6.6)

$$
\begin{aligned}
\frac{\partial v_{\varepsilon}(x)}{\partial x_{j}} & =\frac{1}{\varepsilon^{N}} \int_{\mathbb{R}^{N}} \frac{\partial}{\partial x_{j}}\left\{\eta\left(\frac{y-x}{\varepsilon}, x\right)\right\} \cdot \bar{v}(y) d y \\
& =\frac{1}{\varepsilon^{N}} \int_{\mathbb{R}^{N}}\left\{-\frac{1}{\varepsilon} \frac{\partial \eta}{\partial a_{j}}\left(\frac{y-x}{\varepsilon}, x\right)+\frac{\partial \eta}{\partial b_{j}}\left(\frac{y-x}{\varepsilon}, x\right)\right\} \cdot \bar{v}(y) d y \\
& =\frac{1}{\varepsilon^{N}} \int_{\mathbb{R}^{N}}\left\{-\frac{\partial \eta}{\partial y_{j}}\left(\frac{y-x}{\varepsilon}, x\right)\right\} \cdot \bar{v}(y) d y+\frac{1}{\varepsilon^{N}} \int_{\mathbb{R}^{N}} \frac{\partial \eta}{\partial b_{j}}\left(\frac{y-x}{\varepsilon}, x\right) \cdot \bar{v}(y) d y \\
& =\frac{1}{\varepsilon^{N}} \int_{\mathbb{R}^{N}} \eta\left(\frac{y-x}{\varepsilon}, x\right) \cdot \frac{\partial \bar{v}(y)}{\partial y_{j}} d y+\frac{1}{\varepsilon^{N}} \int_{\mathbb{R}^{N}} \frac{\partial \eta}{\partial b_{j}}\left(\frac{y-x}{\varepsilon}, x\right) \cdot \bar{v}(y) d y \\
& =\int_{\mathbb{R}^{N}} \eta(z, x) \cdot \frac{\partial \bar{v}(x+\varepsilon z)}{\partial x_{j}} d z+\int_{\mathbb{R}^{N}} \frac{\partial \eta}{\partial x_{j}}(z, x) \cdot \bar{v}(x+\varepsilon z) d z \\
& =\boldsymbol{\psi}_{\varepsilon, j}(x)+\int_{\mathbb{R}^{N}} \frac{\partial \eta}{\partial x_{j}}(z, x) \cdot \bar{v}(x+\varepsilon z) d z \\
& =\boldsymbol{\psi}_{\varepsilon, j}(x)+\int_{\mathbb{R}^{N}} \frac{\partial \eta}{\partial x_{j}}(z, x) \cdot(\bar{v}(x+\varepsilon z)-\bar{v}(x)) d z,
\end{aligned}
$$

where $a=\left(a_{1}, \ldots, a_{N}\right)$ and $b=\left(b_{1}, \ldots, b_{N}\right)$ are the first and the second arguments of the function $\eta(a, b): \mathbb{R}^{N} \times \mathbb{R}^{N} \rightarrow \mathbb{R}^{d \times d}$. In the last equality, we have used the fact that $\int_{\mathbb{R}^{N}} \frac{\partial \eta(z, x)}{\partial x_{j}} d z=0$ for every $x \in \Omega$. Differentiating once more gives

$$
\text { (6.7) } \begin{aligned}
& \frac{\partial^{2}}{\partial x_{k} \partial x_{j}} v_{\varepsilon}(x) \\
= & \frac{\partial \boldsymbol{\psi}_{\varepsilon, j}(x)}{\partial x_{k}}+\frac{\partial}{\partial x_{k}}\left(\frac{1}{\varepsilon^{N}} \int_{\mathbb{R}^{N}} \frac{\partial \eta}{\partial b_{j}}\left(\frac{y-x}{\varepsilon}, x\right) \cdot \bar{v}(y) d y\right) \\
= & \frac{\partial \boldsymbol{\psi}_{\varepsilon, j}(x)}{\partial x_{k}}+\left(\frac{1}{\varepsilon^{N}} \int_{\mathbb{R}^{N}} \frac{\partial}{\partial x_{k}}\left\{\frac{\partial \eta}{\partial b_{j}}\left(\frac{y-x}{\varepsilon}, x\right)\right\} \cdot \bar{v}(y) d y\right) \\
= & \frac{\partial \boldsymbol{\psi}_{\varepsilon, j}(x)}{\partial x_{k}}+\frac{1}{\varepsilon^{N}} \int_{\mathbb{R}^{N}}\left(-\frac{1}{\varepsilon} \frac{\partial}{\partial a_{k}}\left\{\frac{\partial \eta}{\partial b_{j}}\left(\frac{y-x}{\varepsilon}, x\right)\right\}+\frac{\partial}{\partial b_{k}}\left\{\frac{\partial \eta}{\partial b_{j}}\left(\frac{y-x}{\varepsilon}, x\right)\right\}\right) \cdot \bar{v}(y) d y \\
= & \frac{1}{\varepsilon^{N}} \int_{\mathbb{R}^{N}}\left(-\frac{\partial}{\partial y_{k}}\left\{\frac{\partial \eta}{\partial b_{j}}\left(\frac{y-x}{\varepsilon}, x\right)\right\}\right) \cdot \bar{v}(y) d y \\
& +\frac{1}{\varepsilon^{N}} \int_{\mathbb{R}^{N}} \frac{\partial}{\partial b_{k}}\left\{\frac{\partial \eta}{\partial b_{j}}\left(\frac{y-x}{\varepsilon}, x\right)\right\} \cdot \bar{v}(y) d y+\frac{\partial \boldsymbol{\psi}_{\varepsilon, j}(x)}{\partial x_{k}} \\
= & \frac{1}{\varepsilon^{N}} \int_{\mathbb{R}^{N}} \frac{\partial \eta}{\partial b_{k}}\left(\frac{y-x}{\varepsilon}, x\right) \cdot \frac{\partial \bar{v}(y)}{\partial y_{j}} d y+\frac{1}{\varepsilon^{N}} \int_{\mathbb{R}^{N}} \frac{\partial^{2} \eta}{\partial b_{k} \partial b_{j}}\left(\frac{y-x}{\varepsilon}, x\right) \cdot \bar{v}(y) d y+\frac{\partial \boldsymbol{\psi}_{\varepsilon, j}(x)}{\partial x_{k}} \\
= & \frac{\partial \boldsymbol{\psi}_{\varepsilon, j}(x)}{\partial x_{k}}+\int_{\mathbb{R}^{N}} \frac{\partial \eta}{\partial x_{k}}(z, x) \cdot \overline{\boldsymbol{\varphi}}_{j}(x+\varepsilon z) d z+\int_{\mathbb{R}^{N}} \frac{\partial^{2} \eta}{\partial x_{k} \partial x_{j}}(z, x) \cdot \bar{v}(x+\varepsilon z) d z \\
= & \frac{\partial \boldsymbol{\psi}_{\varepsilon, j}(x)}{\partial x_{k}}+\int_{\mathbb{R}^{N}} \frac{\partial \eta}{\partial x_{k}}(z, x) \cdot\left(\overline{\boldsymbol{\varphi}}_{j}(x+\varepsilon z)-\overline{\boldsymbol{\varphi}}_{j}(x)\right) d z \\
& +\int_{\mathbb{R}^{N}} \frac{\partial^{2} \eta}{\partial x_{k} \partial x_{j}}(z, x) \cdot(\bar{v}(x+\varepsilon z)-\bar{v}(x)) d z .
\end{aligned}
$$


Therefore,

$$
\begin{gathered}
\left|\nabla^{2} v_{\varepsilon}(x)-\nabla \psi_{\varepsilon}(x)\right| \leq C \quad \text { for } x \in \Omega \text { and all } \varepsilon \\
\lim _{\varepsilon \rightarrow 0}\left|\nabla^{2} v_{\varepsilon}(x)-\nabla \psi_{\varepsilon}(x)\right|=0 \quad \text { for a.e } x \text { in } \Omega,
\end{gathered}
$$

where $C>0$ does not depend on $\varepsilon$. Clearly,

$$
\begin{aligned}
& F\left(a_{2}, b_{2}, c, d\right)-F\left(a_{1}, b_{1}, c, d\right)= \\
& \qquad \begin{array}{l}
\left(\int_{0}^{1} \nabla_{a} F\left(a_{1}+t\left(a_{2}-a_{1}\right), b_{1}+t\left(b_{2}-b_{1}\right), c, d\right) d t\right) \cdot\left(a_{2}-a_{1}\right) \\
\quad+\left(\int_{0}^{1} \nabla_{b} F\left(a_{1}+t\left(a_{2}-a_{1}\right), b_{1}+t\left(b_{2}-b_{1}\right), c, d\right) d t\right) \cdot\left(b_{2}-b_{1}\right)
\end{array}
\end{aligned}
$$

Therefore,

$$
\begin{aligned}
& F\left(\varepsilon \nabla^{2} v_{\varepsilon}, \nabla v_{\varepsilon}, v_{\varepsilon}, f\right)-F\left(\varepsilon \nabla \psi_{\varepsilon}, \psi_{\varepsilon}, v_{\varepsilon}, f\right)= \\
& \begin{aligned}
\varepsilon\left(\int_{0}^{1} \nabla_{a} F\left(\varepsilon \nabla \psi_{\varepsilon}+t\left(\varepsilon \nabla^{2} v_{\varepsilon}-\varepsilon \nabla \psi_{\varepsilon}\right), \psi_{\varepsilon}+t\left(\nabla v_{\varepsilon}-\psi_{\varepsilon}\right), v_{\varepsilon}, f\right) d t\right) \\
\times\left(\nabla^{2} v_{\varepsilon}-\nabla \psi_{\varepsilon}\right)+ \\
\left(\int_{0}^{1} \nabla_{b} F\left(\varepsilon \nabla \psi_{\varepsilon}+t\left(\varepsilon \nabla^{2} v_{\varepsilon}-\varepsilon \nabla \psi_{\varepsilon}\right), \psi_{\varepsilon}+t\left(\nabla v_{\varepsilon}-\psi_{\varepsilon}\right), v_{\varepsilon}, f\right) d t\right) \\
\times\left(\nabla v_{\varepsilon}-\psi_{\varepsilon}\right) .
\end{aligned}
\end{aligned}
$$

Since $\bar{v}$ is a Lipschitz function, we have

$$
\int_{\mathbb{R}^{N}}\left|\nabla_{x} \eta(z, x)\right||\bar{v}(x+\varepsilon z)-\bar{v}(x)| d z \leq C \varepsilon
$$

for some constant $C>0$. Then from (6.6), (6.7), (6.8) and (6.9), we conclude

$$
\begin{aligned}
& \int_{\Omega} \frac{1}{\varepsilon}\left|F\left(\varepsilon \nabla^{2} v_{\varepsilon}, \nabla v_{\varepsilon}, v_{\varepsilon}, f\right)-F\left(\varepsilon \nabla \psi_{\varepsilon}, \psi_{\varepsilon}, v_{\varepsilon}, f\right)\right| d x \leq \\
& \bar{C} \int_{\Omega}\left|\int_{0}^{1} \nabla_{a} F\left(\varepsilon \nabla \psi_{\varepsilon}+t\left(\varepsilon \nabla^{2} v_{\varepsilon}-\varepsilon \nabla \psi_{\varepsilon}\right), \psi_{\varepsilon}+t\left(\nabla v_{\varepsilon}-\psi_{\varepsilon}\right), v_{\varepsilon}, f\right) d t\right| \\
&+\left|\int_{0}^{1} \nabla_{b} F\left(\varepsilon \nabla \psi_{\varepsilon}+t\left(\varepsilon \nabla^{2} v_{\varepsilon}-\varepsilon \nabla \psi_{\varepsilon}\right), \psi_{\varepsilon}+t\left(\nabla v_{\varepsilon}-\psi_{\varepsilon}\right), v_{\varepsilon}, f\right) d t\right| d x \\
&=o_{\varepsilon}(1),
\end{aligned}
$$

where in the last equality we have used the assumption $\nabla_{a} F(0, \varphi, v, f)=0$ and $\nabla_{b} F(\nabla \varphi, \varphi, v, f)=0$ a.e. (because $F(0, \varphi, v, f)=0$ and $F \geq 0$ ). So we get (6.5). 
It remains to prove that

(6.11) $\lim _{\varepsilon \rightarrow 0} \frac{1}{\varepsilon} \int_{\Omega} F\left(\varepsilon \nabla \psi_{\varepsilon}(x), \psi_{\varepsilon}(x), v_{\varepsilon}(x), f(x)\right) d x$

$+\int_{J_{\nabla v}}\left\{\int_{-\infty}^{0} F\left(\left\{p(t, x) \cdot\left(\varphi^{+}(x)-\varphi^{-}(x)\right)\right\} \otimes \boldsymbol{\nu}(x), \Gamma(t, x), v(x), f^{+}(x)\right) d t\right.$

$\left.+\int_{0}^{\infty} F\left(\left\{p(t, x) \cdot\left(\varphi^{+}(x)-\varphi^{-}(x)\right)\right\} \otimes \boldsymbol{\nu}(x), \Gamma(t, x), v(x), f^{-}(x)\right) d t\right\} d \mathcal{H}^{N-1}(x)$,

with

$$
\Gamma(t, x):=\left(\int_{-\infty}^{t} p(s, x) d s\right) \cdot \varphi^{-}(x)+\left(\int_{t}^{\infty} p(s, x) d s\right) \cdot \varphi^{+}(x) .
$$

But this is a direct consequence of Theorem 4.1.

Proof of Theorem 6.2. $\quad$ Since $\widetilde{Y}(\eta)$ and $\widetilde{\mathcal{J}}_{0}(\varphi)$ do not depend on the orientation of the vector $\boldsymbol{\nu}(x)$, we may assume, as before, that the orientation of $J_{\nabla v}$ is such that the function $\nu: J_{\nabla v} \rightarrow S^{N-1}$ is Borel measurable (see [3, Proposition 3.69]). Because $\nabla v \in B V$, we have

$$
\nabla v^{+}(x)-\nabla v^{-}(x)=\chi(x) \otimes \boldsymbol{\nu}(x) .
$$

Thus, we can rewrite (6.4) as

$$
\begin{aligned}
& \tilde{Y}(\eta)= \\
& \int_{J_{\nabla v}}\left\{\int_{-\infty}^{0} F\left(p(t, \cdot) \cdot \chi \otimes \boldsymbol{\nu} \otimes \boldsymbol{\nu}, \int_{t}^{+\infty} p(s, \cdot) d s \cdot \chi \otimes \boldsymbol{\nu}+\nabla v^{-}, v, f^{+}\right) d t\right. \\
& \left.+\int_{0}^{\infty} F\left(p(t, \cdot) \cdot \chi \otimes \boldsymbol{\nu} \otimes \boldsymbol{\nu}, \int_{t}^{+\infty} p(s, \cdot) d s \cdot \chi \otimes \boldsymbol{\nu}+\nabla v^{-}, v, f^{-}\right) d t\right\} d \mathcal{H}^{N-1} .
\end{aligned}
$$

Then we can apply Lemma 5.2 with $\mu:=\left|\nabla v^{+}-\nabla v^{-}\right| \mathcal{H}^{N-1}\left\llcorner J_{\nabla v}, \nu_{0}(x):=\boldsymbol{\nu}(x)\right.$, $h(x):=\left(\nabla v^{+}(x), \nabla v^{-}(x), \chi(x), v(x), f^{+}(x), f^{-}(x), \boldsymbol{\nu}(x)\right)$ to infer

$$
\begin{aligned}
& \inf _{\eta \in \mathcal{V}} \tilde{Y}(\eta)= \\
& \int_{J_{\nabla v}}\left(\operatorname { i n f } _ { p ( t ) \in \mathcal { P } } \left\{\int_{-\infty}^{0} F\left(p(t) \cdot \chi \otimes \boldsymbol{\nu} \otimes \boldsymbol{\nu}, \int_{t}^{+\infty} p(s) d s \cdot \chi \otimes \boldsymbol{\nu}+\nabla v^{-}, v, f^{+}\right) d t\right.\right. \\
& \left.\left.+\int_{0}^{\infty} F\left(p(t) \cdot \chi \otimes \boldsymbol{\nu} \otimes \boldsymbol{\nu}, \int_{t}^{+\infty} p(s) d s \cdot \chi \otimes \boldsymbol{\nu}+\nabla v^{-}, v, f^{-}\right) d t\right\}\right) d \mathcal{H}^{N-1}
\end{aligned}
$$

Finally, Lemma 5.3 gives the desired result. 


\section{Apendix A}

Proof of Lemma 5.1. The lemma is achieved by using several regularization techniques and classical diagonal arguments.

Step 1: Let $\Omega^{\prime} \subset \mathbb{R}^{N}$ be some bounded domain such that $\bar{\Omega} \subset \subset \Omega^{\prime}$. We prove that there exists a Borel measurable function $\eta: \mathbb{R}^{N} \times \mathbb{R}^{N} \rightarrow \mathbb{R}^{d \times d}$ satisfying the following hypotheses:

(i) There exists $\bar{M}>0$ such that $\|\eta\|_{L^{\infty}} \leq \bar{M}$ and $\operatorname{supp} \eta \subset \overline{B_{\bar{M}}(0)} \times \overline{\Omega^{\prime}}$ is compact.

(ii) $\int_{\mathbb{R}^{N}} \eta(z, x) d z=I$ for all $x \in \Omega^{\prime}$.

(iii) $\int_{H_{\nu_{0}(x)}^{0}} \eta\left(t \boldsymbol{\nu}_{0}(x)+y, x\right) d \mathcal{H}^{N-1}(y)=p(t, x)$ for all $x \in \Omega$.

Since $p(t, x) \in \mathcal{W}$, there exists $M>0$ such that $\|p\|_{L^{\infty}} \leq M$ and $\operatorname{supp} p(t, x) \subset$ $B_{M}(0) \times \Omega$. Let $H:[0, \infty) \rightarrow[0, \infty)$ be any continuous function supported in $[0, M]$ satisfying $\int_{\mathbb{R}^{N-1}} H(|x|) d x=1$ if $N>1$ and $H(0)=1$ if $N=1$. Consider also a function $l \in C_{c}\left(\mathbb{R}^{N}, \mathbb{R}^{d \times d}\right)$ satisfying $\int_{\mathbb{R}^{N}} l(z) d z=I$ and $l(z)=0$ for $|z|>M$. Define the function $\eta(z, x): \mathbb{R}^{N} \times \mathbb{R}^{N} \rightarrow \mathbb{R}^{d \times d}$ by

$$
\eta(z, x)= \begin{cases}H\left(\sqrt{|z|^{2}-\left(\boldsymbol{\nu}_{0}(x) \cdot z\right)^{2}}\right) p\left(\boldsymbol{\nu}_{0}(x) \cdot z, x\right), & (z, x) \in \mathbb{R}^{N} \times \Omega, \\ l(z), & (z, x) \in \mathbb{R}^{N} \times\left(\Omega^{\prime} \backslash \Omega\right), \\ 0, & (z, x) \in \mathbb{R}^{N} \times\left(\mathbb{R}^{N} \backslash \Omega^{\prime}\right) .\end{cases}
$$

Then $\eta$ is Borel measurable. Moreover, $\eta$ satisfies hypotheses (i)-(iii).

Step 2: Let $\bar{\mu}$ be the measure on $\mathbb{R}^{N}$ defined by

$$
\bar{\mu}(A)=\mu(A \cap \Omega)+\mathcal{L}^{N}(A) \quad \text { for every Borel set } A \subset \mathbb{R}^{N} .
$$

Consider a bounded domain $\Omega^{\prime \prime}$, such that $\Omega \subset \subset \Omega^{\prime \prime} \subset \subset \Omega^{\prime}$. We prove that there exists a sequence $\left\{\hat{\eta}_{n}\right\} \subset C_{c}\left(\mathbb{R}^{N} \times \mathbb{R}^{N}, \mathbb{R}^{d \times d}\right)$, such that

(i') $\int_{\mathbb{R}^{N}} \hat{\eta}_{n}(z, x) d z=I$ for every $x \in \overline{\Omega^{\prime \prime}}$ and there exists $\tilde{M}>0$, independent of $n$, such that supp $\hat{\eta}_{n} \subset \overline{B_{\tilde{M}}(0)} \times \mathbb{R}^{N}$ and $\left\|\hat{\eta}_{n}\right\|_{L^{\infty}} \leq \tilde{M}$.

(ii') $\lim _{n \rightarrow \infty} \int_{\mathbb{R}^{N}} \int_{\mathbb{R}^{N}}\left|\hat{\eta}_{n}(z, x)-\eta(z, x)\right| d z d \bar{\mu}(x)=0$.

This clearly implies

$$
\lim _{n \rightarrow \infty} \int_{\Omega} \int_{\mathbb{R}}\left|\hat{p}_{n}(t, x)-p(t, x)\right| d t d \mu(x)=0,
$$

where

$$
\hat{p}_{n}(t, x)=\int_{H_{\nu_{0}(x)}^{0}} \hat{\eta}_{n}\left(t \boldsymbol{\nu}_{0}(x)+y, x\right) d \mathcal{H}^{N-1}(y) .
$$


For every $0<r<1, z \in \mathbb{R}^{N}$ and $x \in \mathbb{R}^{N}$ define

$$
\eta_{0, r}(z, x):=\frac{1}{\mathcal{L}^{N}\left(B_{r}(z)\right)} \int_{B_{r}(z)} \eta(y, x) d y=\frac{1}{\mathcal{L}^{N}\left(B_{r}(0)\right)} \int_{B_{r}(0)} \eta(z+y, x) d y .
$$

Then $\eta_{0, r}$ is a Borel measurable function which satisfies

$$
\left|\eta_{0, r}\left(z_{1}, x\right)-\eta_{0, r}\left(z_{2}, x\right)\right| \leq C_{r}\left|z_{1}-z_{2}\right| \quad \text { for all } z_{1}, z_{2} \in \mathbb{R}^{N} \text { and } x \in \mathbb{R}^{N},
$$

where $C_{r}$ depends only on $r$. We also have $\left|\eta_{0, r}(z, x)\right| \leq \bar{M}$ for all $z$ and $x$ and $\operatorname{supp} \eta_{0, r} \subset \overline{B_{M+1}(0)} \times \overline{\Omega^{\prime}}$. For each $x \in \Omega^{\prime}$,

$$
\begin{aligned}
\int_{\mathbb{R}^{N}} \eta_{0, r}(z, x) d z & =\frac{1}{\mathcal{L}^{N}\left(B_{r}(0)\right)} \int_{\mathbb{R}^{N}}\left(\int_{B_{r}(0)} \eta(z+y, x) d y\right) d z \\
& =\frac{1}{\mathcal{L}^{N}\left(B_{r}(0)\right)} \int_{B_{r}(0)}\left(\int_{\mathbb{R}^{N}} \eta(z, x) d z\right) d y=I .
\end{aligned}
$$

From Theorem 1 in [13, Section 1.7], we infer that for any fixed $x \in \mathbb{R}^{N}$,

$$
\lim _{r \rightarrow 0^{+}} \eta_{0, r}(z, x)=\eta(z, x), \quad \text { for a.e. } z \in \mathbb{R}^{N} .
$$

Take a sequence $r_{n} \downarrow 0$ and define a sequence of functions $\left\{\bar{\eta}_{n}(z, x)\right\}$ on $\mathbb{R}^{N} \times \mathbb{R}^{N}$ by $\bar{\eta}_{n}(z, x)=\eta_{0, r_{n}}(z, x)$. Clearly, $\left\|\bar{\eta}_{n}\right\|_{L^{\infty}} \leq \bar{M}$ and supp $\bar{\eta}_{n} \subset \overline{B_{M+1}(0)} \times \overline{\Omega^{\prime}}$. By (A.2),

$$
\lim _{n \rightarrow \infty} \int_{\mathbb{R}^{N}} \int_{\mathbb{R}^{N}}\left|\bar{\eta}_{n}(z, x)-\eta(z, x)\right| d z d \bar{\mu}(x)=0 .
$$

In addition, for all $x \in \mathbb{R}^{N}$ and $z_{1}, z_{2} \in \mathbb{R}^{N}$,

$$
\left|\bar{\eta}_{n}\left(z_{1}, x\right)-\bar{\eta}_{n}\left(z_{2}, x\right)\right| \leq C_{n}\left|z_{1}-z_{2}\right|,
$$

where $C_{n}>0$ depends only on $n$.

For every $n$ and $\rho>0$, define $\eta_{n, \rho}: \mathbb{R}^{N} \times \mathbb{R}^{N} \rightarrow \mathbb{R}$ by

$$
\eta_{n, \rho}(z, x)=\frac{1}{\bar{\mu}\left(B_{\rho}(x)\right)} \int_{B_{\rho}(x)} \bar{\eta}_{n}(z, y) d \bar{\mu}(y) .
$$

Then, for $\rho>0$ sufficiently small and any $x \in \Omega^{\prime \prime}$, we have

$$
\int_{\mathbb{R}^{N}} \eta_{n, \rho}(z, x) d z=\frac{1}{\bar{\mu}\left(B_{\rho}(x)\right)} \int_{B_{\rho}(x)} \int_{\mathbb{R}^{N}} \bar{\eta}_{n}(z, y) d z d \bar{\mu}(y)=I .
$$

Using (A.4), we obtain

(A.5) $\left|\eta_{n, \rho}\left(z_{1}, x_{1}\right)-\eta_{n, \rho}\left(z_{2}, x_{2}\right)\right| \leq\left|\eta_{n, \rho}\left(z_{1}, x_{1}\right)-\eta_{n, \rho}\left(z_{1}, x_{2}\right)\right|+C_{n}\left|z_{1}-z_{2}\right|$. 
But, for every $z \in \mathbb{R}^{N}$, we have

$$
\begin{aligned}
\mid \int_{B_{\rho}\left(x_{1}\right)} \bar{\eta}_{n}(z, y) d \bar{\mu}(y) & -\int_{B_{\rho}\left(x_{2}\right)} \bar{\eta}_{n}(z, y) d \bar{\mu}(y) \mid \\
\leq & \bar{M}\left\{\bar{\mu}\left(B_{\rho}\left(x_{1}\right) \backslash B_{\rho}\left(x_{2}\right)\right)+\bar{\mu}\left(B_{\rho}\left(x_{1}\right) \backslash B_{\rho}\left(x_{2}\right)\right)\right\} .
\end{aligned}
$$

Therefore, combining (A.5) with (A.6) we get that $\eta_{n, \rho}$ is continuous on $\mathbb{R}^{N} \times \mathbb{R}^{N}$. Again, by Theorem 1 in [13, Section 1.7], for any fixed $z \in \mathbb{R}$,

$$
\lim _{\rho \rightarrow 0^{+}} \eta_{n, \rho}(z, x)=\bar{\eta}_{n}(z, x), \quad \text { for } \bar{\mu} \text {-almost every } x \in \mathbb{R}^{N} \text {. }
$$

We also have

- $\left\|\eta_{n, \rho}\right\|_{L^{\infty}} \leq \bar{M}$,

- $\operatorname{supp} \eta_{n, \rho} \subset \overline{B_{M+1}(0)} \times \mathbb{R}^{N}$,

- there exists $\bar{M}_{0}>0$ such that for $\rho>0$ sufficiently small and any $n$, $\operatorname{supp} \eta_{n, \rho} \subset \mathbb{R}^{N} \times \overline{B_{M_{0}}(0)}$.

By (A.7), we obtain

$$
\lim _{\rho \rightarrow 0^{+}} \int_{\mathbb{R}^{N}} \int_{\mathbb{R}^{N}}\left|\eta_{n, \rho}(z, x)-\bar{\eta}_{n}(z, x)\right| d z d \bar{\mu}(x)=0 .
$$

It follows from (A.3) that there exists a sequence $\rho_{n} \downarrow 0$ such that

$$
\lim _{n \rightarrow \infty} \int_{\mathbb{R}^{N}} \int_{\mathbb{R}^{N}}\left|\eta_{n, \rho_{n}}(z, x)-\eta(z, x)\right| d z d \bar{\mu}(x)=0,
$$

and $\int_{\mathbb{R}^{N}} \eta_{n, \rho_{n}}(z, x) d z=I$ for every $n$ and each $x \in \overline{\Omega^{\prime \prime}}$. Put $\hat{\eta}_{n}(z, x):=\eta_{n, \rho_{n}}(z, x)$. Then the sequence $\left\{\hat{\eta}_{n}\right\}$ satisfies $\left(\mathrm{i}^{\prime}\right)$ and $\left(\mathrm{ii}^{\prime}\right)$.

Step 3: We are ready to complete the proof. Let $\omega \in C_{c}^{\infty}\left(\mathbb{R}^{N} \times \mathbb{R}^{N}\right)$ be such that $\omega \geq 0$ and $\int_{\mathbb{R}^{N}} \int_{\mathbb{R}^{N}} \omega\left(\zeta_{1}, \zeta_{2}\right) d \zeta_{1} d \zeta_{2}=1$. For any $0<\varepsilon<1$, define

$$
\begin{aligned}
\left(\eta_{n}\right)_{\varepsilon}(z, x) & =\frac{1}{\varepsilon^{2 N}} \int_{\mathbb{R}^{N}} \int_{\mathbb{R}^{N}} \omega\left(\frac{y_{1}-z}{\varepsilon}, \frac{y_{2}-x}{\varepsilon}\right) \hat{\eta}_{n}\left(y_{1}, y_{2}\right) d y_{1} d y_{2} \\
& =\int_{\mathbb{R}^{N}} \int_{\mathbb{R}^{N}} \omega\left(\zeta_{1}, \zeta_{2}\right) \hat{\eta}_{n}\left(z+\varepsilon \zeta_{1}, x+\varepsilon \zeta_{2}\right) d \zeta_{1} d \zeta_{2} .
\end{aligned}
$$

Then $\left(\eta_{n}\right)_{\varepsilon} \in C_{c}^{2}\left(\mathbb{R}^{N} \times \mathbb{R}^{N}, \mathbb{R}^{d \times d}\right)$ and there exists $\hat{M}>0$, independent of $n$ and $\varepsilon$, such that for every $0<\varepsilon<1$ and every $n$, we have $\operatorname{supp}\left(\eta_{n}\right)_{\varepsilon} \subset \overline{B_{\hat{M}}(0)} \times \mathbb{R}^{N}$ and $\left\|\left(\eta_{n}\right)_{\varepsilon}\right\|_{L^{\infty}} \leq \hat{M}$. Moreover, for sufficiently small $\varepsilon>0$, we have

$$
\begin{aligned}
& \int_{\mathbb{R}^{N}}\left(\eta_{n}\right)_{\varepsilon}(z, x) d z \\
& =\frac{1}{\varepsilon^{2 N}} \int_{\mathbb{R}^{N}}\left(\int_{\mathbb{R}^{N}} \int_{\mathbb{R}^{N}} \omega\left(y_{1} / \varepsilon, y_{2} / \varepsilon\right) \hat{\eta}_{n}\left(z+y_{1}, x+y_{2}\right) d y_{1} d y_{2}\right) d z \\
& =\frac{1}{\varepsilon^{2 N}} \int_{\mathbb{R}^{N}} \int_{\mathbb{R}^{N}} \omega\left(y_{1} / \varepsilon, y_{2} / \varepsilon\right)\left(\int_{\mathbb{R}^{N}} \hat{\eta}_{n}\left(z+y_{1}, x+y_{2}\right) d z\right) d y_{1} d y_{2}=I \quad \text { for all } x \in \Omega .
\end{aligned}
$$


Therefore, $\left(\eta_{n}\right)_{\varepsilon} \in \mathcal{V}$ for $\varepsilon>0$ sufficiently small. For $t \in \mathbb{R}$ and $x \in \Omega$, set

$$
\left(\hat{p}_{n}\right)_{\varepsilon}(t, x)=\int_{H_{\nu_{0}(x)}^{0}}\left(\eta_{n}\right)_{\varepsilon}\left(t \boldsymbol{\nu}_{0}(x)+y, x\right) d \mathcal{H}^{N-1}(y) .
$$

Since $\left(\eta_{n}\right)_{\varepsilon} \rightarrow \eta_{n}$ uniformly on $\mathbb{R}^{N} \times \mathbb{R}^{N}$ as $\varepsilon \downarrow 0$, we conclude that

$$
\lim _{\varepsilon \rightarrow 0^{+}} \int_{\Omega} \int_{\mathbb{R}}\left|\left(\hat{p}_{n}\right)_{\varepsilon}(t, x)-\hat{p}_{n}(t, x)\right| d t d \mu(x)=0 .
$$

Therefore, by (A.1), there exists a sequence $\varepsilon_{n} \downarrow 0$ such that $\left(\eta_{n}\right)_{\varepsilon_{n}} \in \mathcal{V}$ and

$$
\lim _{n \rightarrow \infty} \int_{\Omega} \int_{\mathbb{R}}\left|\left(\hat{p}_{n}\right)_{\varepsilon_{n}}(t, x)-p(t, x)\right| d t d \mu(x)=0 .
$$

Set $\zeta_{n}(z, x):=\left(\eta_{n}\right)_{\varepsilon_{n}}(z, x) \in \mathcal{V}$, and define $p_{n}(t, x)$ on $\mathbb{R} \times \Omega$ by

$$
p_{n}(t, x)=\int_{H_{\nu_{0}(x)}^{0}} \zeta_{n}\left(t \boldsymbol{\nu}_{0}(x)+y, x\right) d \mathcal{H}^{N-1}(y) .
$$

There exists $C_{0}$ such that $\left|p_{n}(t, x)\right| \leq C_{0}$ for all $x \in \Omega$ and $t \in \mathbb{R}$, and there exists $\hat{C}$ such that $p_{n}(t, x)=0$ for $|t|>\hat{C}$ and any $x \in \Omega$. Moreover, we have

$$
\lim _{n \rightarrow \infty} \int_{\Omega} \int_{\mathbb{R}}\left|p_{n}(t, x)-p(t, x)\right| d t d \mu(x)=0,
$$

so that Lemma 5.1 follows.

\section{REFERENCES}

[1] L. Ambrosio, Metric space valued functions of bounded variation, Ann. Scuola Norm. Sup. Pisa Cl. Sci. (4) 17 (1990), 439-478.

[2] L. Ambrosio, C. De Lellis and C. Mantegazza, Line energies for gradient vector fields in the plane, Calc. Var. Partial Differential Equations 9 (1999), 327-355.

[3] L. Ambrosio, N. Fusco and D. Pallara, Functions of Bounded Variation and Free Discontinuity Problems, Oxford University Press, New York, 2000.

[4] P. Aviles and Y. Giga, A mathematical problem related to the physical theory of liquid crystal configurations, Proc. Centre Math. Anal. Austral. Nat. Univ. 12 (1987), 1-16.

[5] P. Aviles and Y. Giga, On lower semicontinuity of a defect energy obtained by a singular limit of the Ginzburg-Landau type energy for gradient fields, Proc. Roy. Soc. Edinburgh Sect. A 129 (1999), 1-17.

[6] S. Conti and B. Schweizer, A sharp-interface limit for a two-well problem in geometrically linear elasticity. Arch. Ration. Mech. Anal. 179 (2006), 413-452.

[7] S. Conti and B. Schweizer, Rigidity and gamma convergence for solid-solid phase transitions with SO(2)-invariance, Comm. Pure Appl. Math. 59 (2006), 830-868.

[8] S. Conti, I. Fonseca, and G. Leoni, A $\Gamma$-convergence result for the two-gradient theory of phase transitions, Comm. Pure Appl. Math. 55 (2002), 857-936. 
[9] S. Conti and C. De Lellis, Sharp upper bounds for a variational problem with singular perturbation, Math. Ann. 338 (2007), 119-146.

[10] C. De Lellis, An example in the gradient theory of phase transitions ESAIM Control Optim. Calc. Var. 7 (2002), 285-289 (electronic).

[11] A. DeSimone, S. Müller, R.V. Kohn and F. Otto, A compactness result in the gradient theory of phase transitions, Proc. Roy. Soc. Edinburgh Sect. A 131 (2001), 833-844.

[12] L. C. Evans, Partial Differential Equations, American Mathematical Society, Providence, RI, 1998.

[13] L. C. Evans and R.F. Gariepy, Measure Theory and Fine Properties of Functions, CRC Press, Boca Raton, FL, 1992.

[14] I. Fonseca and C. Mantegazza, Second order singular perturbation models for phase transitions, SIAM J. Math. Anal. 31 (2000), 1121-1143 (electronic).

[15] D. Gilbarg and N. Trudinger, Elliptic Partial Differential Equations of Elliptic Type, 2nd ed., Springer-Verlag, Berlin-Heidelberg, 1983.

[16] E. Giusti, Minimal Surfaces and Functions of Bounded Variation, Birkhäuser Verlag, Basel, 1984.

[17] W. Jin and R.V. Kohn, Singular perturbation and the energy of folds, J. Nonlinear Sci. 10 (2000), 355-390.

[18] L. Modica, The gradient theory of phase transitions and the minimal interface criterion, Arch. Rational Mech. Anal. 98 (1987), 123-142.

[19] L. Modica and S. Mortola, Il limite nella $\Gamma$-convergenza di una famiglia di funzionali ellittici, Boll. Un. Mat. Ital. A (5) 14 (1977), 526-529.

[20] L. Modica and S. Mortola, Un esempio di $\Gamma^{-}$-convergenza, Boll. Un. Mat. Ital. B (5) 14 (1977), 285-299.

[21] A. Poliakovsky, A method for establishing upper bounds for singular perturbation problems, C. R. Math. Acad. Sci. Paris 341 (2005), 97-102.

[22] A. Poliakovsky, On a singular perturbation problem related to optimal lifting in BV-space, Calc. Var. Partial Differential Equations, 28 (2007), 411-426.

[23] A. Poliakovsky, Upper bounds for singular perturbation problems involving gradient fields, J. Eur. Math. Soc. 9 (2007), 1-43.

[24] P. Sternberg, The effect of a singular perturbation on nonconvex variational problems, Arch. Rational Mech. Anal. 101 (1988), 209-260.

[25] A. I. Volpert and S. I. Hudjaev, Analysis in Classes of Discontinuous Functions and Equations of Mathematical Physics, Martinus Nijhoff Publishers, Dordrecht, 1985.

\author{
Arkady Poliakovsky \\ INSTITUT FÜR MATHEMATIK, \\ UNIVERSITÄT ZÜRICH \\ WINTERTHURERSTRASSE 190 \\ CH-8057 ZÜRICH, SWITZERLAND \\ email: apoliako@math.unizh.ch
}

(Received December 15, 2006 and in revised form September 21, 2007) 